ORNL/ER-172

Energy Systems Environmental Restoration Program

ORNL Environmental Restoration Program

\title{
Results of the Radiological Characterization Survey of the Building 7819 Decontamination Facility at Oak Ridge National Laboratory, Oak Ridge, Tennessee
}

\author{
R. E. Rodriguez \\ K. S. Brown \\ D. E. Rice \\ R. C. Gosslee \\ V. P. Patania
}

Date Issued-December 1993

Prepared by

Health Sciences Research Division

Oak Ridge National Laboratory

Prepared for

U.S. Department of Energy

Office of Environmental Restoration and Waste Management

Facility Transition and Management Program

under budget and reporting code EW 7010050

OAK RIDGE NATIONAL LABORATORY

Oak Ridge, Tennessee 37831-6285

managed by

MARTIN MARIETTA ENERGY SYSTEMS, INC.

for the

U.S. DEPARTMENT OF ENERGY

under contract DE-AC05-84OR21400 


\section{Author Affiliations}

R. E. Rodriguez, K. S. Brown, D. E. Rice, R. C. Gosslee, and V. P. Patania are members of the Measurement Applications and Development Group of the Health Sciences Research Division, Oak Ridge National Laboratory, Martin Marietta Energy Systems, Inc. 


\section{CONTENTS}

FIGURES $\ldots \ldots \ldots \ldots \ldots \ldots \ldots \ldots \ldots \ldots \ldots \ldots \ldots \ldots \ldots \ldots$

TABLES $\ldots \ldots \ldots \ldots \ldots \ldots \ldots \ldots \ldots \ldots \ldots \ldots \ldots \ldots \ldots \ldots \ldots \ldots \ldots$

ACKNOWLEDGMENTS $\ldots \ldots \ldots \ldots \ldots \ldots \ldots \ldots \ldots \ldots \ldots \ldots \ldots$ ix

EXECUTIVE SUMMARY $\ldots \ldots \ldots \ldots \ldots \ldots \ldots \ldots \ldots \ldots \ldots \ldots \ldots \ldots$

1. INTRODUCTION $\ldots \ldots \ldots \ldots \ldots \ldots \ldots \ldots \ldots \ldots \ldots \ldots \ldots \ldots$

2. RADIOLOGICAL SURVEY METHODS $\ldots \ldots \ldots \ldots \ldots \ldots \ldots \ldots \ldots$

2.1 GAMMA MEASUREMENTS $\ldots \ldots \ldots \ldots \ldots \ldots \ldots \ldots \ldots \ldots \ldots$

2.2 BETA-GAMMA MEASUREMENTS $\ldots \ldots \ldots \ldots \ldots \ldots \ldots \ldots \ldots \ldots$

2.3 ALPHA MEASUREMENTS $\ldots \ldots \ldots \ldots \ldots \ldots \ldots \ldots \ldots \ldots \ldots \ldots$

2.4 NEUTRON MEASUREMENTS $\ldots \ldots \ldots \ldots \ldots \ldots \ldots \ldots \ldots \ldots \ldots \ldots$

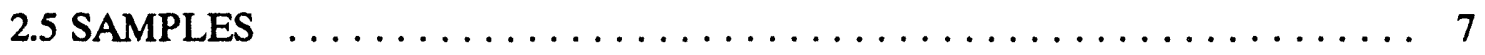

3. SURVEY RESULTS $\ldots \ldots \ldots \ldots \ldots \ldots \ldots \ldots \ldots \ldots \ldots \ldots$

3.1 GENERAL OBSERVATIONS $\ldots \ldots \ldots \ldots \ldots \ldots \ldots \ldots \ldots \ldots \ldots \ldots \ldots$

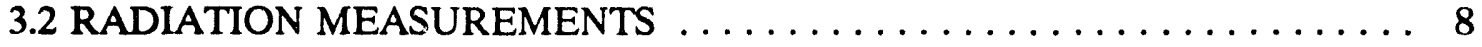

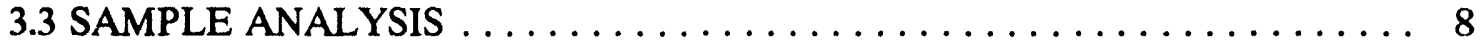

4. SIGNIFICANCE OF FINDINGS $\ldots \ldots \ldots \ldots \ldots \ldots \ldots \ldots \ldots \ldots \ldots$

REFERENCES $\ldots \ldots \ldots \ldots \ldots \ldots \ldots \ldots \ldots \ldots \ldots \ldots \ldots \ldots \ldots \ldots \ldots \ldots$ 


\section{FIGURES}

1 Location of the Building 7819 Decontamination Facility in Waste Area

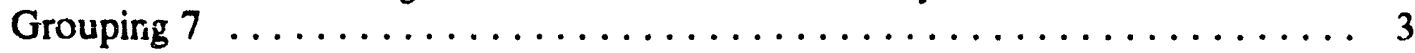

2 View looking northwest at the Building 7819 Decontamination Facility

(July 1993) ........................... 4

3 View looking southwest at the Building 7819 Decontamination Facility ..... 5

4 Evidence of suspect asbestos in northwest corner of Building 7819

(July 1993) ............................ 9

5 Snakeskin on overhead heater in Building 7819 (July 1993) . . . . . . . 10

6 Interior of eastern side of Building 7819 showing pit mill (July 1993) . . . . 11

7 Collection of overhead smea: sample in Building 7819 (July 1993) . . . . . 12

8 Air sampler located on interior wall of Building 7819 (July 1993) . . . . . . 13

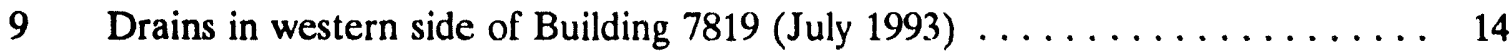

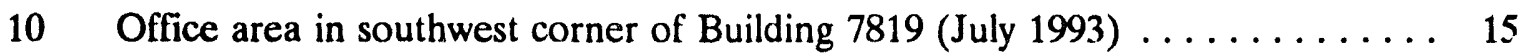

11 Gamma exposure rate $(\mathrm{mR} / \mathrm{h}) 1 \mathrm{~m}$ above the floor in each grid block

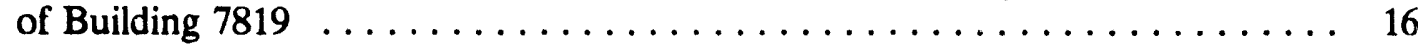

12 View of highly contaminated pump in southeastern end of Building $\mathbf{7 8 1 9}$

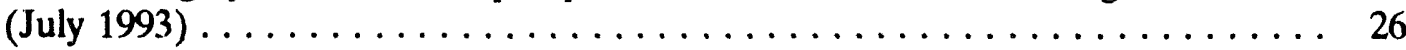

13 View of contaminated metal table in northeastern end of Building 7819 (July 1993) . . . . . . . . . . . . . . . . . . . . . . . . . . . . . . . .

14 View of grossly contaminated ledge above sink in northeastern end of Building 7819 (July 1993) . . . . . . . . . . . . . . . . . . . . 28

15 Sampling locations for transferable alpha and beta-gamma contamination on walls in Building 7819

16 Sampling locations for transferable alpha and beta-gamma contamination

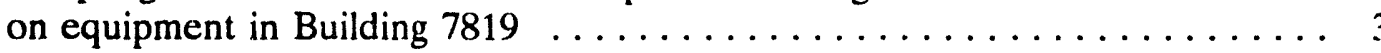

17 Sampling locations for transferable alpha and beta-gamma contamination on

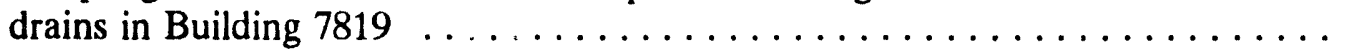

18 Sampling locations for transferable alpha and beta-gamma contamination on overhead surfaces in Building 7819 


\section{TABLES}

1 Summary of radiation measurements on floor of Building 7819

Decontamination Facility . . . . . . . . . . . . . . . . . . . 17

2 Summary of radiation measurements on interior and exterior walls of

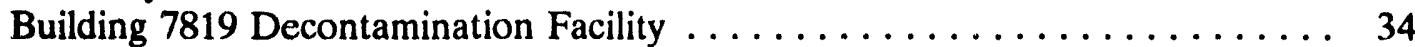

3 Summary of radiation measurements on equipment in Building 7819

Decontamination Facility

4 Summary of radiation measurements on drains in Building 7819

Decontamination Facility

5 Summary of overhead radiation measurements in Building 7819

Decontamination Facility

6 Activity levels of gross alpha, gross beta, ${ }^{90} \mathrm{Sr},{ }^{60} \mathrm{Co}$, and ${ }^{137} \mathrm{Cs}$ in smear samples from Building 7819 Decontamination Facility 


\section{ACKNOWLEDGMENTS}

This project was sponsored by the U.S. Department of Energy's Office of Environmental Restoration and Waste Management Facility Transition and Management Program in support of the Oak Ridge National Laboratory (ORNL) Environmental Restoration Program. The authors wish to acknowledge the support of $K$. Constant and T. W. Burwinkle of the ORNL Waste Management and Remedial Action Division. Measurement Applications and Development (MAD) group member R. E. Rodriguez served as field survey supervisor. The MAD survey team consisted of R. C. Gosslee, D. D. McKinney, V. P. Patania, and D. E. Rice. The authors appreciate the technical and review assistance of W. D. Cottrell, M. E. Murray, M. S. Uziel, and J. K. Williams of the Health Sciences Research Division, A. C. Butler of Geofon Inc., and A. L. Harkey of the Publications Division. The graphics support of S. C. Thompson and J. Lovegrove is also appreciated. 


\section{EXECUTIVE SUMMARY}

A radiological characterization survey of the interior of the Building 7819 Decontamination Facility at Oak Ridge National Laboratory (ORNL) was conducted during July 1993. The investigation was performed by the Measurement Applications and Development Group of the Health Sciences Research Division of ORNL at the request of the Department of Energy Office of Environmental Restoration and Waste Management Facility Transition and Management Program. Building 7819 was in service during the 1960s and 1970s to decontaminate various types of operating equipment and materials in use at ORNL from laboratories and hot cells.

The interior of Building 7819 is grossly contaminated, and the contamination is highly transferable. Levels of alpha and beta contamination inside the building exceed ORNL guidelines for zoning as a Contamination Area. Gamma exposure rates at several locations in the building exceed ORNL guidelines for zoning as a Radiation Area. The survey team measured very high levels of contamination in the northeast corner of the building and lower levels in the southwest corner of the building. Suspected asbestos insulation was observed in the northwest corner of the building on a pipe near the floor. A snakeskin was observed on an overhead heater, indicating the intrusion of wildlife.

Gamma whole-body exposure rates generally ranged from 0.1 to $20 \mathrm{mR} / \mathrm{h}$. Total betagamma surface contamination ranged from 1000 to $520,000 \mathrm{dpm} / 100 \mathrm{~cm}^{2}$, and transferable beta-gamma contamination ranged from 200 to $174,000 \mathrm{dpm} / 100 \mathrm{~cm}^{2}$. A pump and a metal table near the pit exhibited beta dose rates of $3 \mathrm{rad} / \mathrm{h}$ and $300 \mathrm{mrad} / \mathrm{h}$, respectively, and gamma exposure rates of $250 \mathrm{mR} / \mathrm{h}$ and $25 \mathrm{mR} / \mathrm{h}$ at contact, respectively.

Total alpha contamination levels ranged from 100 to $110,000 \mathrm{dpm} / 100 \mathrm{~cm}^{2}$, and transferable contamination ranged from 21 to $440 \mathrm{dpm} / 100 \mathrm{~cm}^{2}$. The ledge at the north end of the pit above the sink demonstrated alpha contamination of approximately $100,000 \mathrm{dpm} / 100 \mathrm{~cm}^{2}$. Radionuclide analysis of 11 smear samples showed the primary beta emitters to be ${ }^{90} \mathrm{Sr}$ and ${ }^{137} \mathrm{Cs}$ with trace amounts of ${ }^{60} \mathrm{Co}$.

The Building 7819 Decontamination Facility was zoned as a Contamination Area and Radiation Area at the time of this survey, and the zoning is justified by the results of the survey. The building will also be zoned as an Airborne Radioactivity Area, based on the results of air sampling.* Recommendations for corrective actions are included.

*M. L. Conner, Office of Radiation Protection, Oak Ridge Natl. Lab., personal communication to K. J. Brown, Health Sciences Research Division, Oak Ridge Natl. Lab., November 1993. 


\section{INTRODUCTION}

A radiological characterization survey of the interior of the Building 7819 Decontamination Facility at Oak Ridge National Laboratory (ORNL) was conducted during July 1993. The investigation was performed by the Measurement Applications and Development Group of the Health Sciences Research Division of ORNL at the request of the Department of Energy (DOE) Office of Environmental Restoration and Waste Management Facility Transition and Management Program.

The Building 7819 Decontamination Facility is located in Waste Area Group (WAG) 7. Building 7819 is shown at the number 7.1 in Fig. 1 (ref. 1). WAG 7 is located in Melton Valley about 1 mile south of the ORNL main plant area. The decontamination facility is located north of Lagoon Road near the entrance to Solid Waste Storage Area (SWSA) 4 at ORNL grid coordinates N19,330 feet and E27,040 feet. Figures 2 and 3 show views of the building looking northwest and southwest.

Frior to construction of the Building 7819 Decontamination Facility, decontamination activities at ORNL were carried out in Building 3036. Wastes were drained to holding tanks under the buildings and transported by truck to what was referred to as the Burial Ground (currently known as SWSA 6) for disposal. Building 7819 was constructed to isolate the decontamination activities from the main plant area and facilitate disposal of the large volumes of liquid waste generated during decontamination activities.

Building 7819 was in service during the 1960 s and 1970 s to decontaminate various forms of operating equipment and materials in use at ORNL from laboratories and hot cells. Examples of items subject to decontamination include electrical tools and isotope carriers of various sizes. Acid baths and abrasive blasting were used in the decontamination process, primarily in the northern section of the building. Liquid wastes were released from Building 7819 into Pit 1 (identified as numeral 7.5 on Fig. 1). Decontamination processes were halted due to concerns about the environmental impact of (1) disposal of large volumes of liquid wastes, and (2) potential runoff from disposal areas in surface water and groundwater.*

The building is currently inactive and unoccupied. Some contaminated equipment is stored inside. The interior of the building is zoned as a Contamination Area. The exterior of the building is surrounded by barbed-wire fencing on three sides and a chain-link fence fronting Lagoon Road with radiation control warning signs at intervals.

The survey included the following:

- measurement of direct gamma radiation at $1 \mathrm{~m}$ above the floor,

- a survey for total alpha contamination,

*A. C. Butler, Geofon Inc., personal communication to K. J. Brown, Health Sciences Research Division, Oak Ridge Natl. Lab., October 1993. 
- a limited survey for fixed beta-gamma contamination,

- collection of one air sample to be counted for gross alpha and gross beta contamination,

- determination of potential dose rates from exposure to neutrons, and

- assessment of possible nonradiological hazards such as suspected asbestos material. 


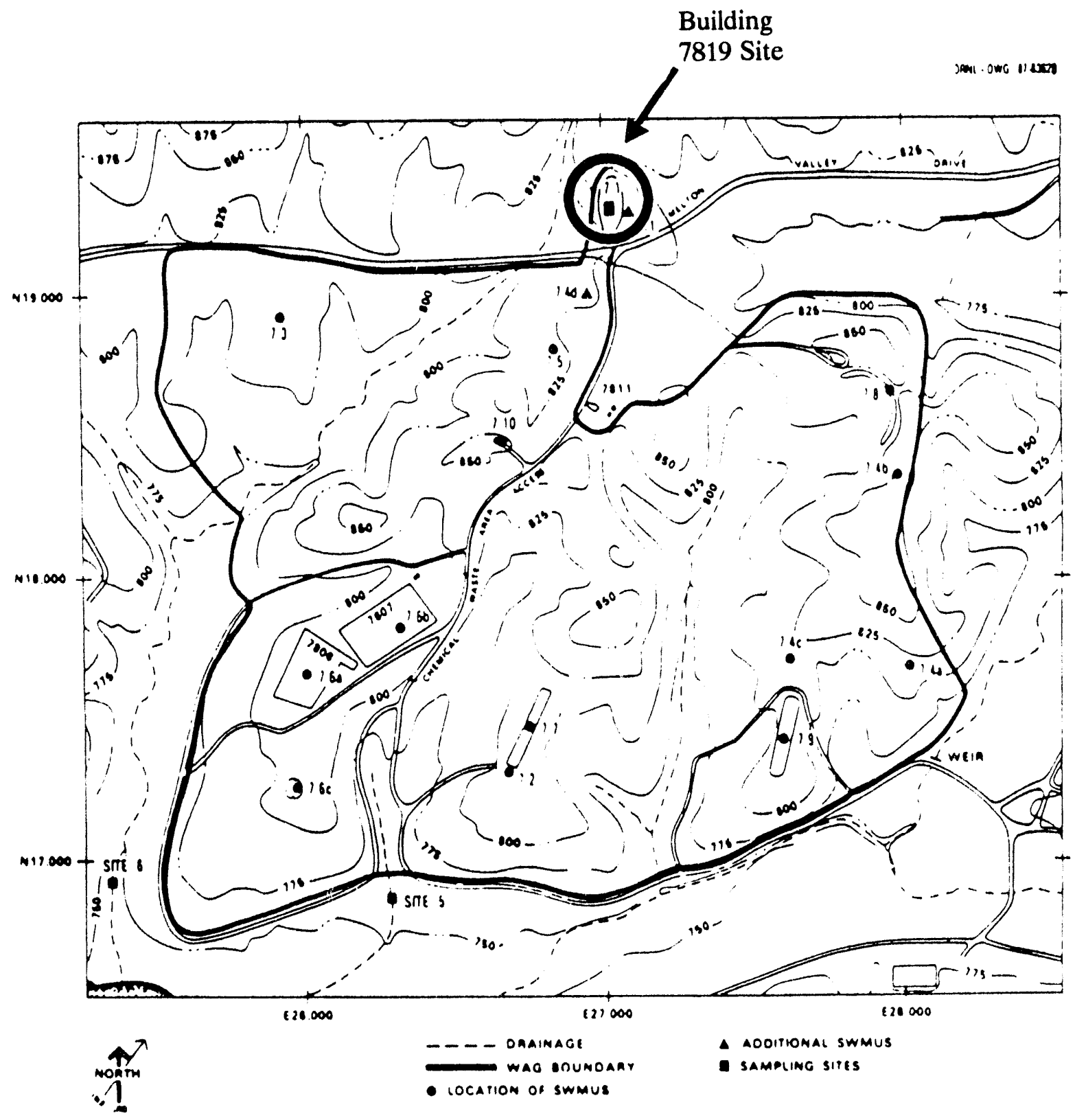

Fig. 1. Location of the Building 7819 Decontamination Facility in Waste Area Grouping 7. 
ORNL Photo 5948-93

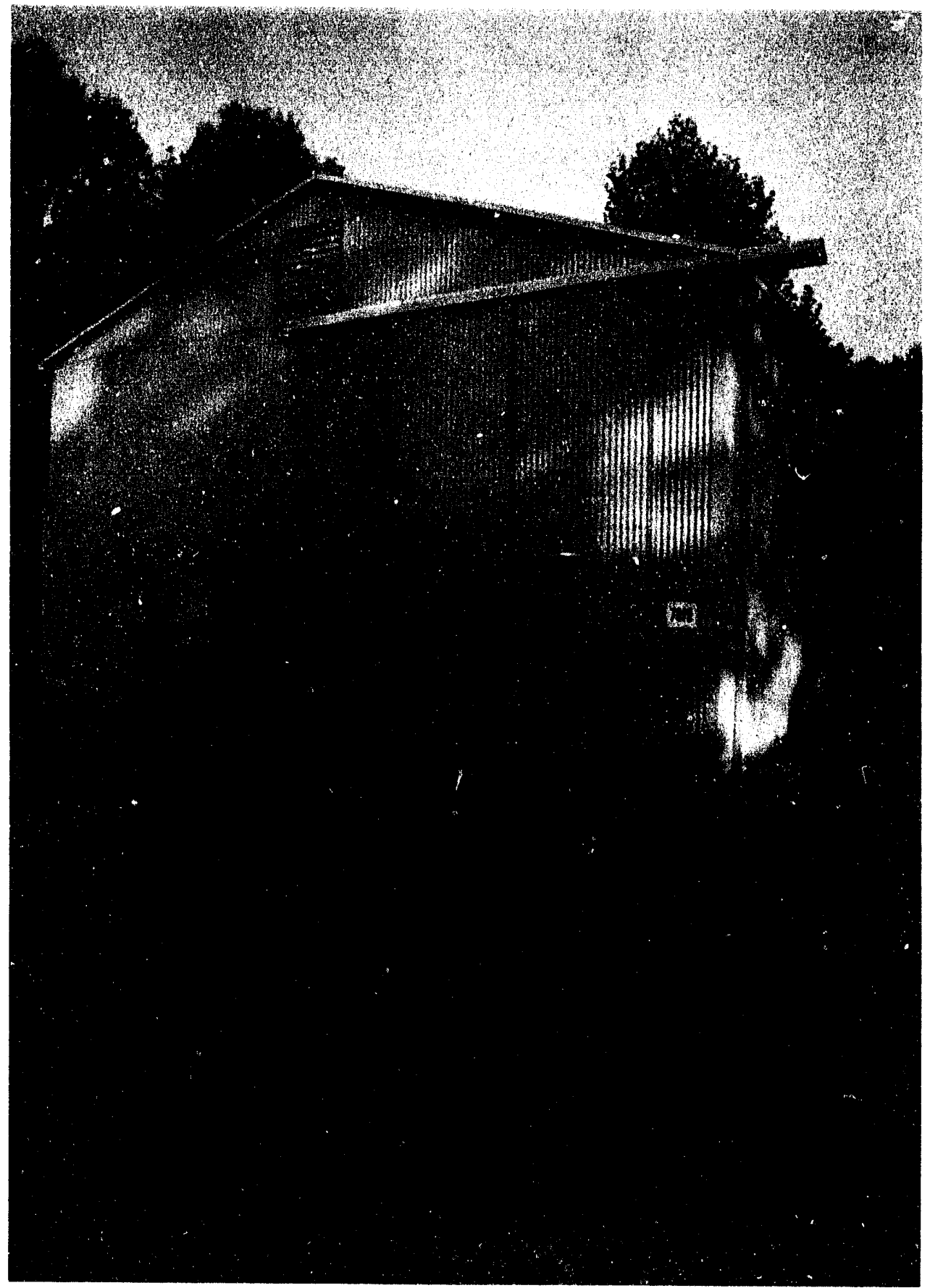

Fig. 2 View looking northwest at the Building 7819 Decontamination Facility (July 1993). 


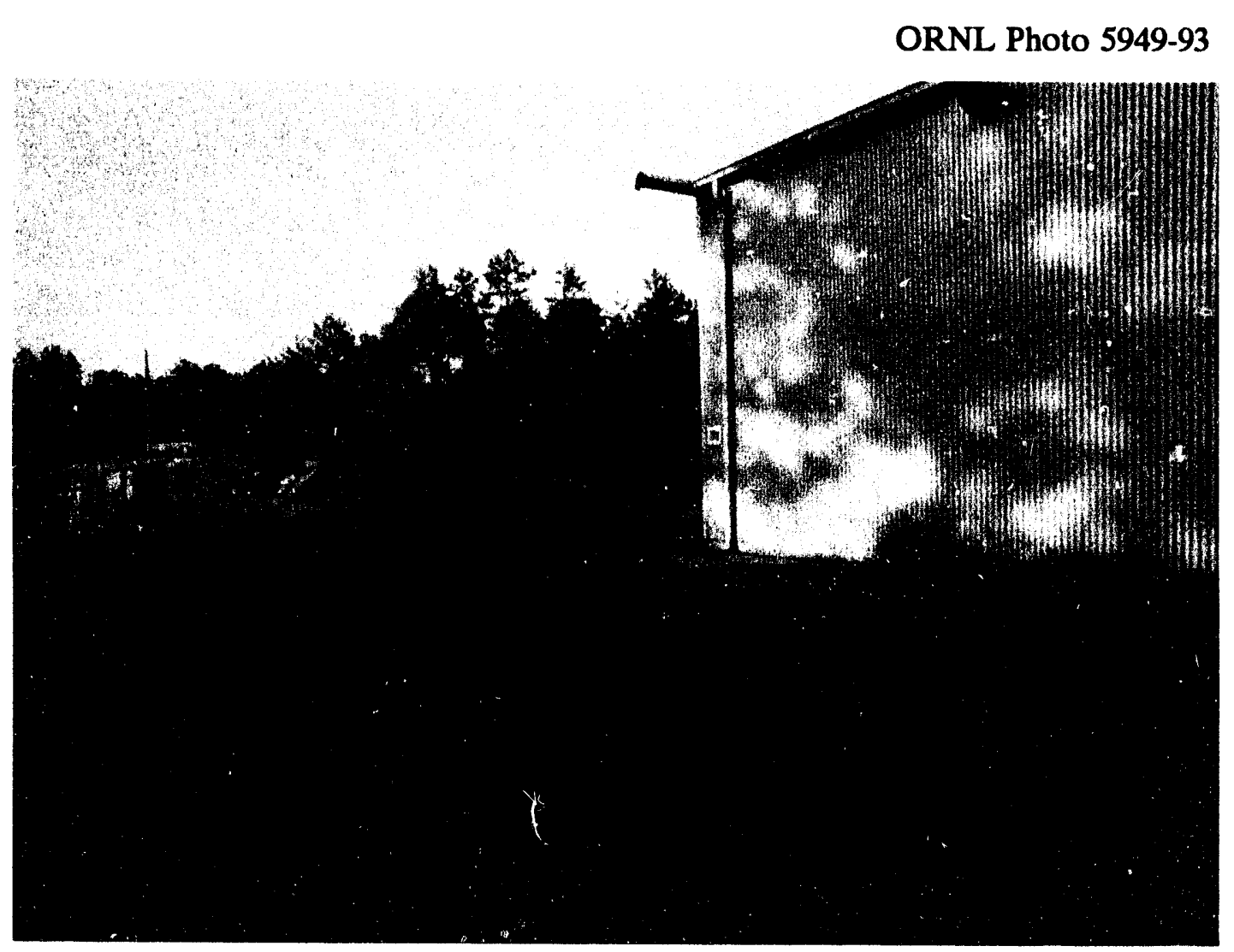

Fig. 3. View looking southwest at the Building 7819 Decontamination Facility (July 1993). 


\section{RADIOLOGICAL SURVEY METHODS}

A description of typical methods and instrumentation providing guidance for the conduct of this survey is presented in Procedures Manual for the ORNL Radiological Survey Activities (RASA) Program, ORNL/TM-8600 (April 1987)'.

Prior to exiting the site, survey personnel were checked for alpha and beta-gamma contamination. A Bicron Analyst meter with an ORNL zinc su'fide (ZnS) alpha scintillator probe was used to check for alpha contamination. A Bicron Analyst meter with an HP-260 probe ("pancake") was used to check for beta-gamma contamination.

\subsection{GAMMA MEASUREMENTS}

Gamma radiation exposure rates were measured with a Bicron Analyst RSO-5 ionization chamber (standard model) throughout the building. Measurements were taken in the center of each $1-\mathrm{m}$ grid block $1 \mathrm{~m}$ above the floor and in contact with selected equipment in the building.

\subsection{BETA-GAMMA MEASUREMENTS}

Beta-gamma radiation levels were measured in each accessible floor grid block using a portable Bicron Analyst scaler/ratemeter with an Eberline HP-210 Geiger-Mueller pancake detector ( $<2 \mathrm{mg} / \mathrm{cm}^{2}$ wall thickness) with fine mesh screen. One-minute counts were taken in the middle of each floor grid. In some areas elevated radiation levels due to shine* from material in the building prevented determination of total beta contamination. In other areas the beta radiation levels were so high that it was necessary to use an RSO-5 ionization chamber instrument and measure only beta radiation dose rates. A smear sample was taken from the floor in each accessible grid block to determine transferable beta-gamma contamination.

Building walls were partially scanned for beta-gamma contamination, because shine from surrounding objects prevented determination of total beta-gamma contamination in some wall sections, and other sections were inaccessible. Transferable beta-gamma contamination on selected walls, equipment, floor drains, and overhead surfaces was assessed by taking smear samples.

*The term shine refers to gamma radiation emanating from a source or sources extrinsic to the area being defined. This extraneous radiation may increase gamma radiation levels above actual levels associated with the area of interest. 


\section{ALPHA MEASUREMENTS}

Alpha radiation was measured in each accessible floor grid block using a portable Bicron Analyst alpha survey meter with a Bicron Analyst A50 "bear-claw" type ZnS scintillation probe. Counts per minute were recorded for a 60 -s direct measurement and converted to disintegrations per minute $(\mathrm{dpm})$ per $100 \mathrm{~cm}^{2}$ using an instrument-specific conversion factor. Personnel, equipment, and smear sample containers were monitored for alpha contamination.

\section{NEUTRON MEASUREMENTS}

An NRC RemRad NP-A2 ("Snoopy") fast neutron meter was used to determine potential fast neutron dose rates in selected areas.

\section{SAMPLES}

Smear samples were collected at systematic locations on floors and walls for measurement of transferable contamination. In addition, smear samples were collected from selected equipment and drains at locations of elevated radiation levels, and from overhead beams and ledges, for determination of transferable alpha and beta contamination. Selected smear samples from the building interior and equipment were sent to the ORNL Analytical Chemistry Division for radionuclide analysis.

An area air sample was obtained in Building 7819 while survey work was being conducted. The air sampler was placed in grid block F,3 at a height of $5 \mathrm{ft} 2$ in. and sampled a volume of $112 \mathrm{ft}^{3}$ in $160 \mathrm{~min}$. The air was then filtered through an $0.8-\mu \mathrm{m}$ millipore fitter. The filter was counted on smear counters for gross alpha and gross beta contamination. 


\section{SURVEY RESULTS}

\subsection{GENERAL OBSERVATIONS}

The interior of Building 7819 is grossly contaminated, and the contamination is extremely transferable. Levels of contamination inside the building exceed ORNL guidelines for alpha and beta contamination. The survey team measured very high levels of contamination in the northeast corner of the building and lower levels in the southwest corner of the building. The telephone on the north wall is contaminated. Dust and oil were found on the floor. Cobwebs and dust were observed throughout the building. Lead bricks and boxes were found, in addition to bagged material (waste) bearing radiation tags from 1978 stating "Highly alpha contaminated." Most drains are plugged. Suspected asbestos was observed in the northwest corner of the building on a pipe near the floor (see Fig. 4). A snakeskin was observed on an overhead heater (Fig. 5), indicating the intrusion of wildlife at one time. Figs. 6-10 are photos of the interior of Building 7819 showing surveying procedures, the general condition of the building, and areas of elevated radioactivity.

\subsection{RADIATION MEASUREMENTS}

Gamma exposure rates inside the building exceeded ORNL guidelines for posting as a Radiation Area in several locations. Gamma whole-body exposure rates generally ranged from 0.1 to $20 \mathrm{mR} / \mathrm{h}$. Figure 11 and Table 1 indicate the gamma exposure rate $(\mathrm{mR} / \mathrm{h}) 1 \mathrm{~m}$ above the floor in each grid block of Building 7819.

Total beta-gamma surface contamination ranged from 1000 to $520,000 \mathrm{dpm} / 100 \mathrm{~cm}^{2}$, and transferable beta-gamma contamination ranged from 200 to $174,000 \mathrm{dpm} / 100 \mathrm{~cm}^{2}$. A pump occupying grid blocks $D, 7$ and $D, 8$ demonstrated a beta dose rate of $3 \mathrm{rad} / \mathrm{h}$ and a gamma exposure rate of $250 \mathrm{mR} / \mathrm{h}$ at contact (Fig. 12). A metal table in grid block L,7 showed a beta dose rate of $300 \mathrm{mrad} / \mathrm{h}$ and a gamma exposure rate of $25 \mathrm{mR} / \mathrm{h}$ at contact (Fig. 13). Table 1 shows total surface beta-gamma contamination and transferable beta-gamma contamination in disintegrations/minute $/ 100 \mathrm{~cm}^{2}$ in each grid block of the floor of Building 7819 .

Alpha contamination was detected in several locations. Total contamination levels ranged from 100 to $110,000 \mathrm{dpm} / 100 \mathrm{~cm}^{2}$, and transferable contamination ranged from 21 to 440 $\mathrm{dpm} / 100 \mathrm{~cm}^{2}$. The ledge at the north end of the pit above the sink indicated alpha contamination of approximately $100,000 \mathrm{dpm} / 100 \mathrm{~cm}^{2}$ (Fig. 14). Table 1 shows total surface alpha contamination and transferable alpha contamination in disintegrations/minute $/ 100 \mathrm{~cm}^{2}$ in each grid block of the floor of Building 7819.

Neutron radiation dose rates were less than $0.2 \mathrm{mrem} / \mathrm{h}$.

\subsection{SAMPLE ANALYSIS}

Sampling locations for transferable alpha and beta-gamma contamination are shown in Fig. 15 for samples taken from the walls and in Fig. 16 for samples taken from equipment. 
ORNL Photo 5977-93

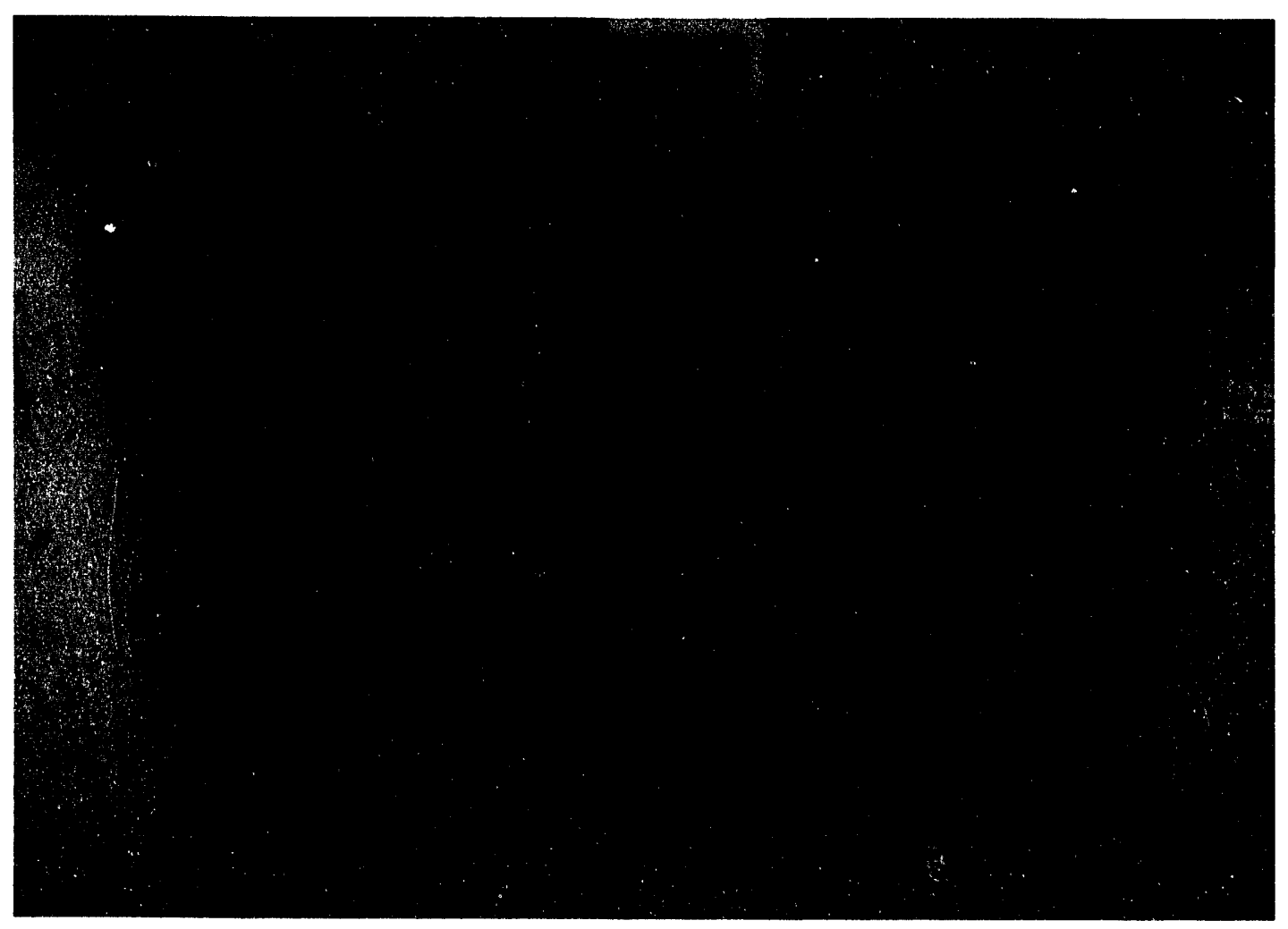

Fig. 4. Evidence of suspect asbestos in northwest corner of Building 7819 (July 1993). 
ORNL Photo 5967-93

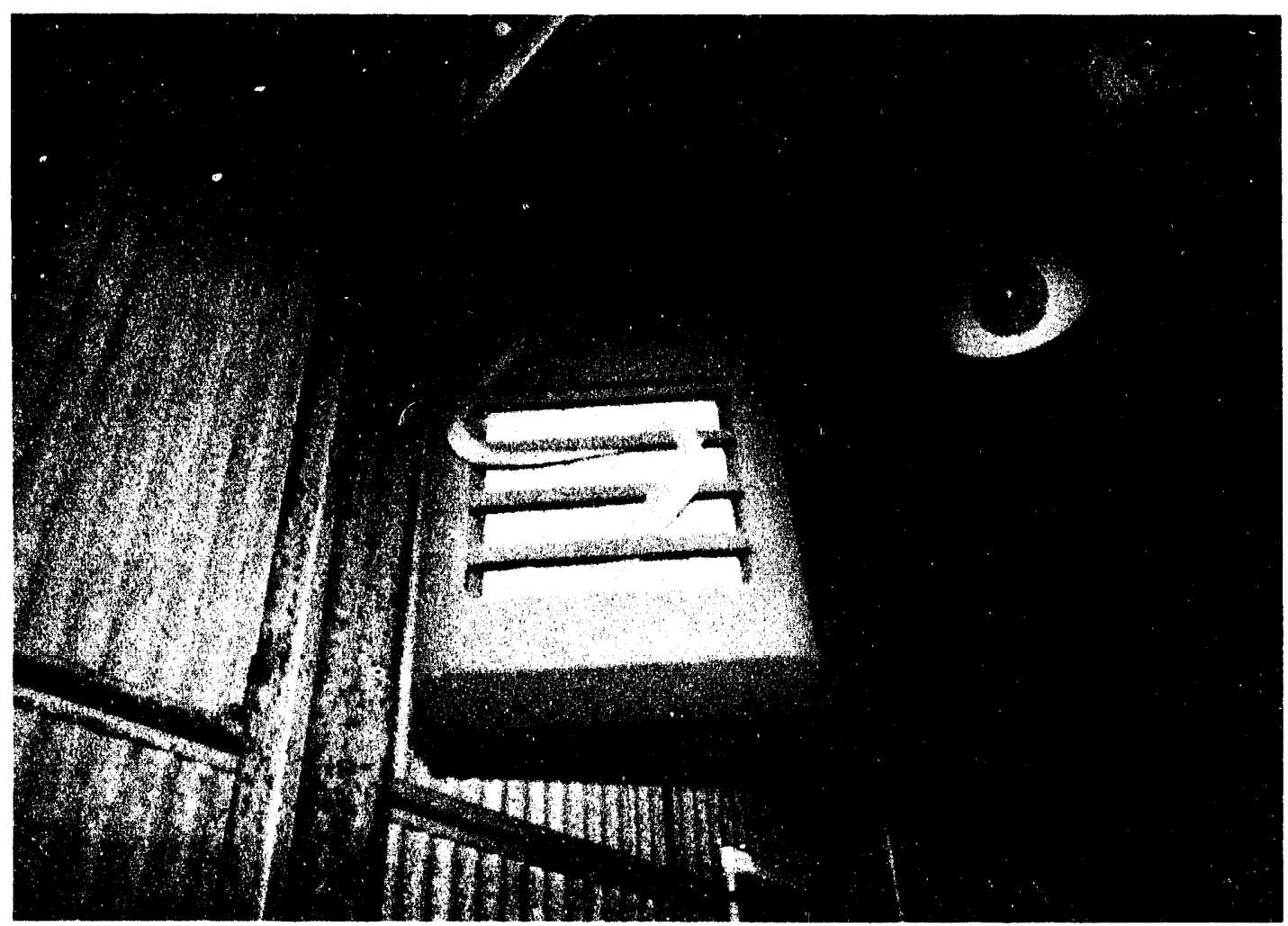

Fig. 5. Snakeskin on overhead heater in Building 7819 (July 1993). 
ORNL Photo 5978-93

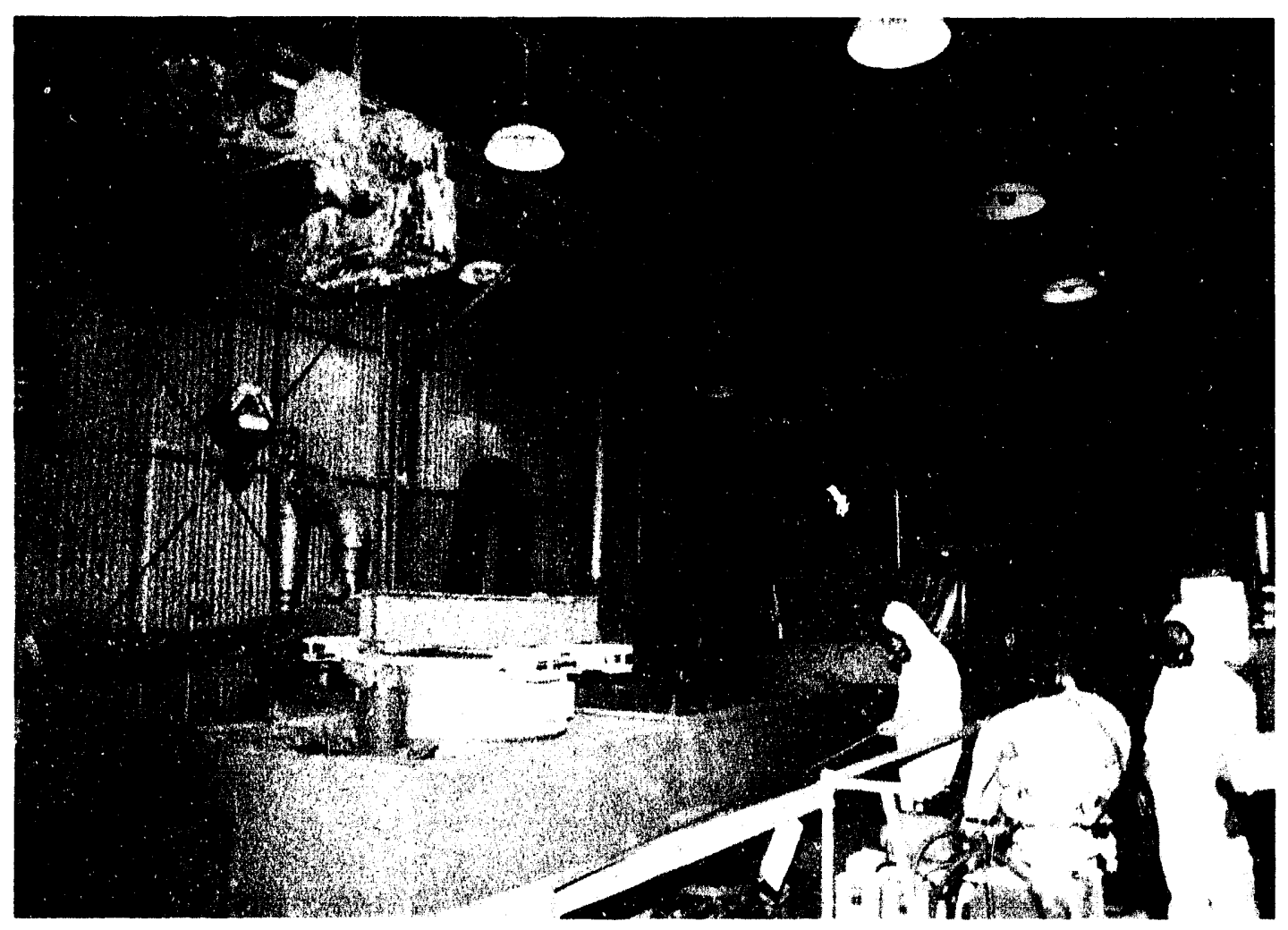

Fig. 6. Interior of eastern side of Building 7819 showing pit mill (July 1993). 
ORNL Photo 5955-93

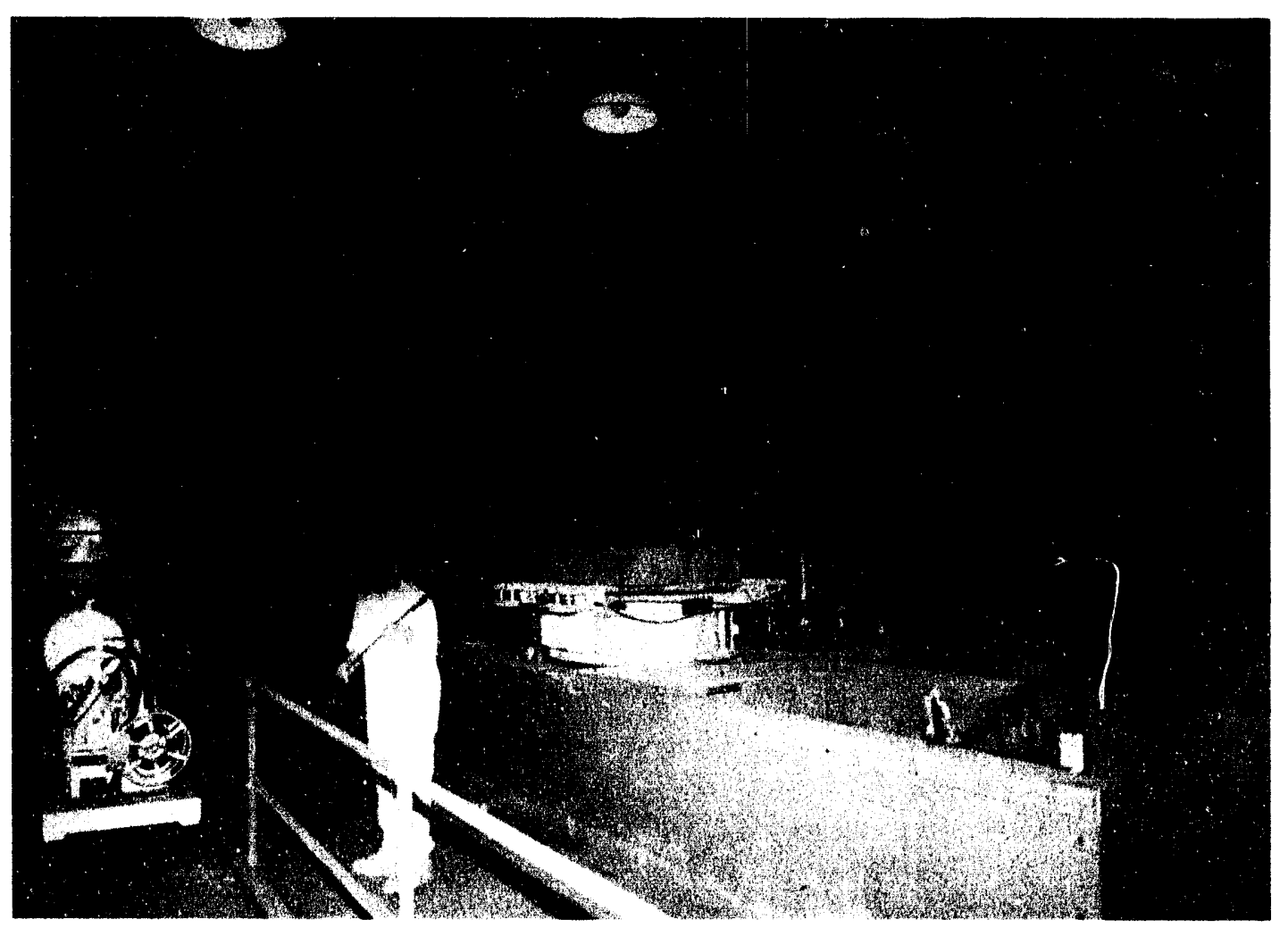

Fig. 7. Collection of overhead smear samples in Building 7819 (July 1993). 
ORNL Photo 5979-93

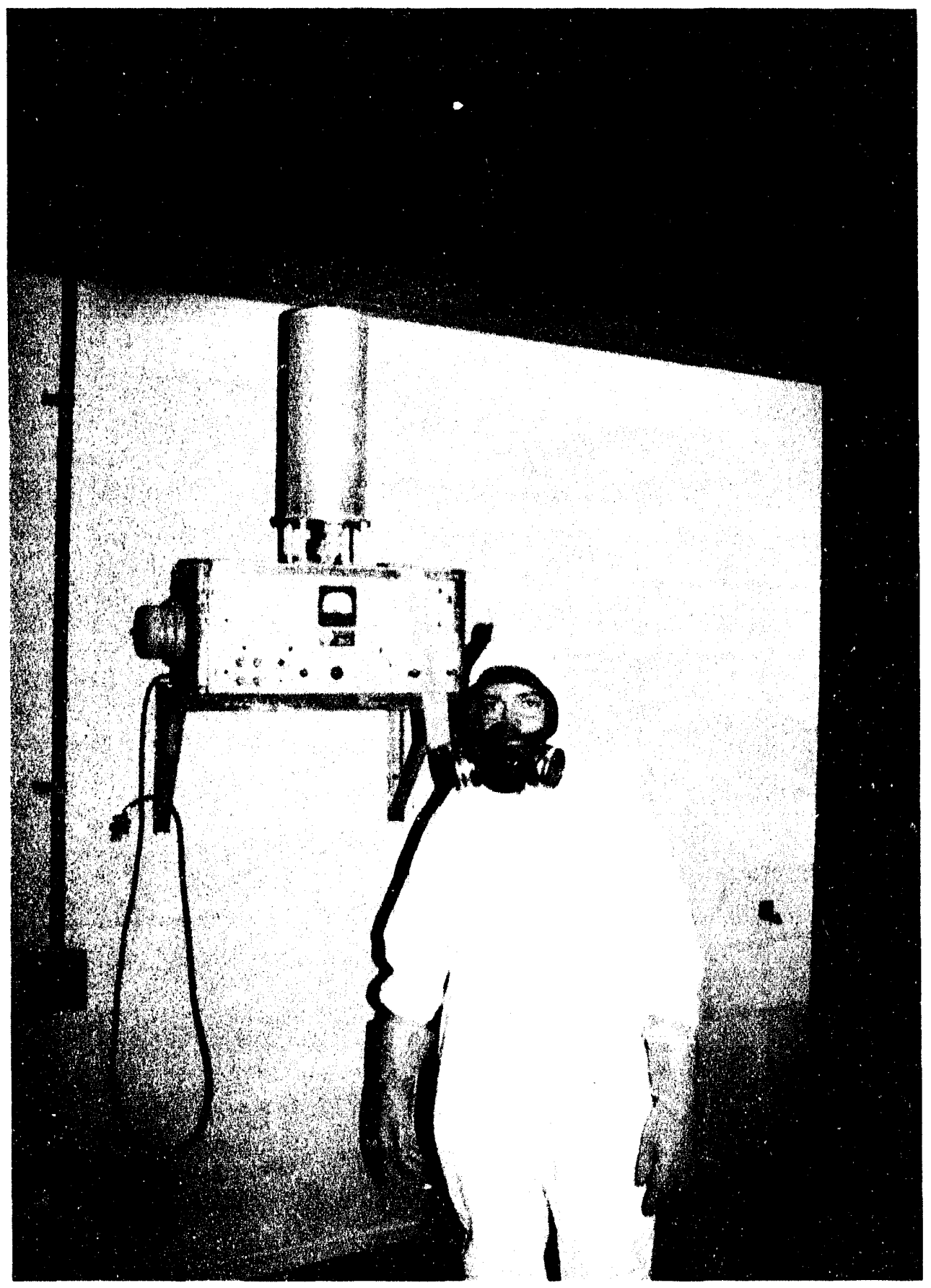

Fig. 8. Air sampler located on interior wall of Building 7819 (July 1993). 
ORNL Photo 5973-93

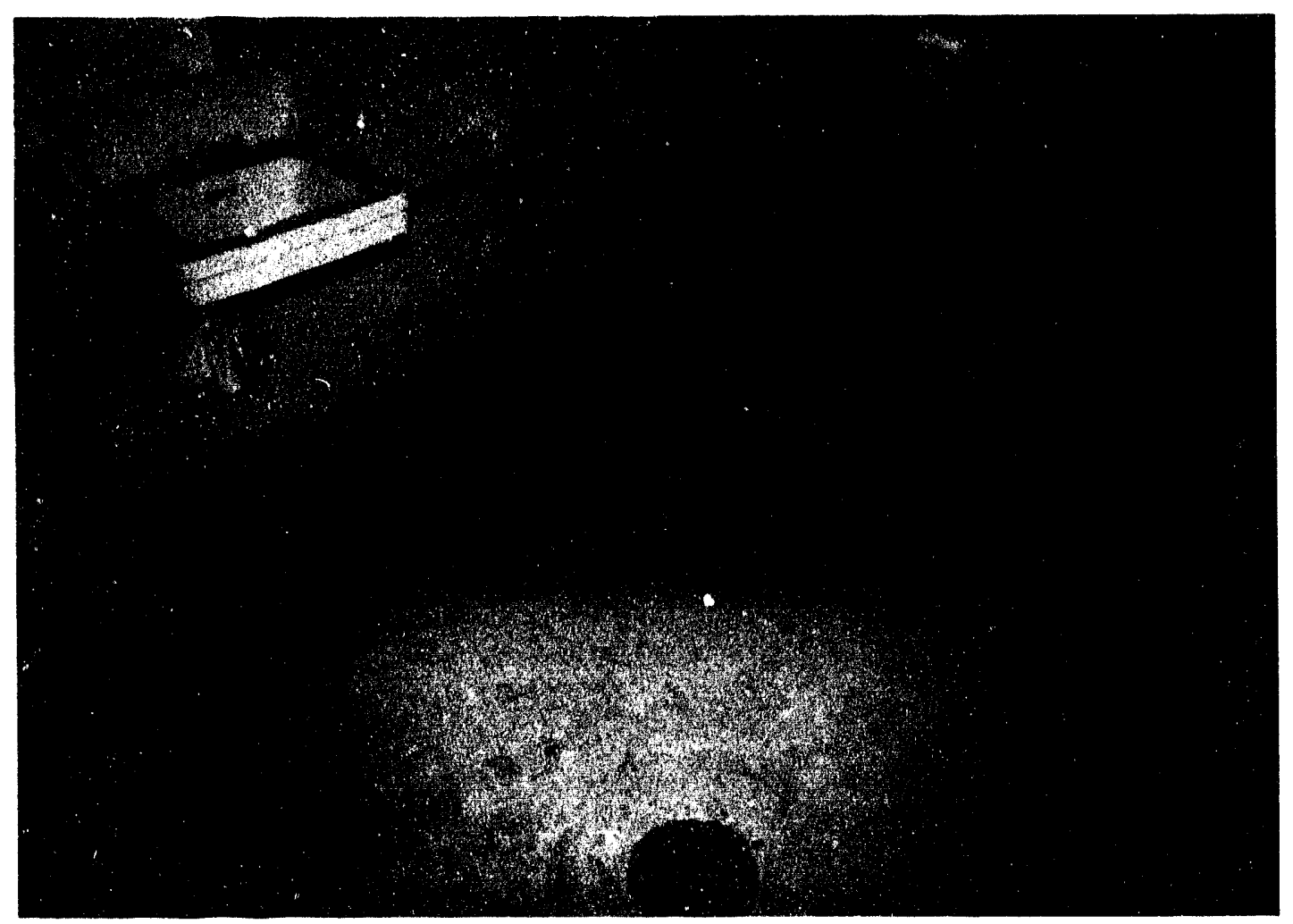

Fig. 9. Drains in western side of Building 7819 (July 1993). 
ORNL Photo 5956-93

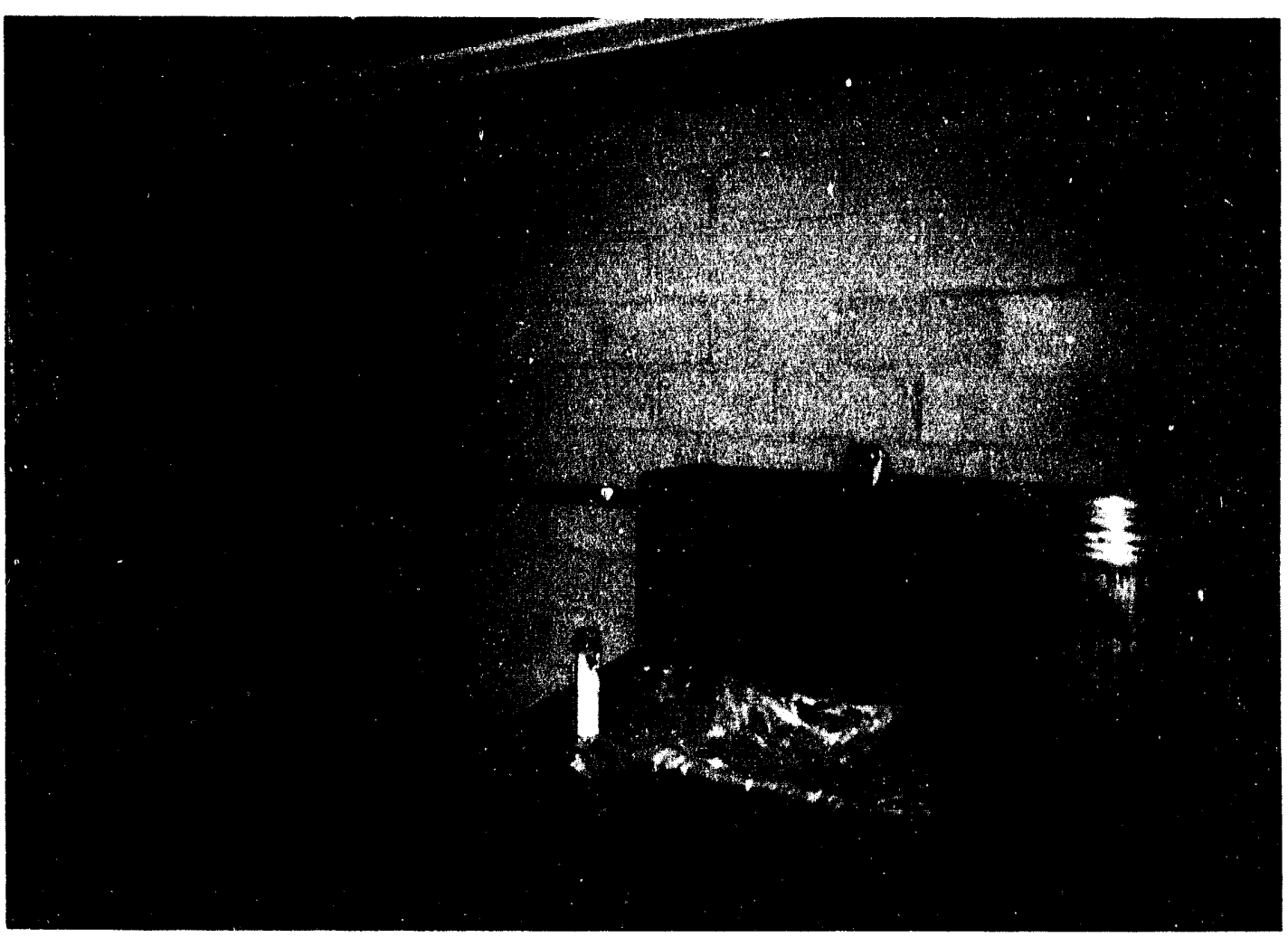

Fig. 10. Office area in southwest corner of Building 7819 (July 1993). 
* = INACCESSIBLE

\begin{tabular}{|c|c|c|c|c|c|c|c|c|c|c|}
\hline \multirow{2}{*}{0} & 0.3 & 0.3 & 0.3 & 0.3 & * & $*$ & 0.1 & 0.5 & 0.8 & 0.7 \\
\hline & 1.7 & 0.8 & 0.4 & 0.3 & 0.2 & 0.2 & 0.6 & 0.8 & 1.7 & 1.0 \\
\hline $\mathrm{N}$ & 4.8 & 1.5 & 1.0 & 0.5 & 0.5 & 0.4 & 0.6 & 1.2 & 2.6 & 1.2 \\
\hline M & 1.1 & 0.4 & 0.3 & 0.3 & 0.5 & 0.5 & 0.8 & 1.4 & 1.7 & 0.8 \\
\hline L & 0.5 & 0,6 & 0.4 & 0.3 & 0.4 & 0.5 & 1.5 & 20.0 & 1.3 & 0.7 \\
\hline K & 0.4 & 0.4 & 0.3 & 0.3 & 0.4 & 0.6 & 0.8 & & * & $*$ \\
\hline & 0.2 & 0.2 & 0.3 & 0.4 & 0.5 & 0.7 & 1.4 & $\star$ & * & * \\
\hline \multirow[t]{2}{*}{1} & & & & & & & & & & \\
\hline & 0.2 & 0.2 & 0.4 & 0.4 & 0.5 & 0.7 & 1.2 & * & * & * \\
\hline \multirow[t]{2}{*}{$\mathrm{H}$} & 2 & & & & & & & & & \\
\hline & 0.2 & 0.2 & 0.2 & 0.6 & 0.7 & 0.8 & 0.8 & * & * & $*$ \\
\hline G & 0.1 & 0.1 & 0.2 & 0.7 & 0.9 & 1.0 & 1.5 & * & * & * \\
\hline $\boldsymbol{F}$ & $<0.1$ & $<0.1$ & $<0.1$ & 0.7 & 0.8 & 1.8 & 2.6 & 4.3 & 3.4 & 2.8 \\
\hline E & 0.0 & 0.1 & 0.0 & 0.6 & 0.8 & 1.5 & 4.4 & 10.0 & 5.0 & 3.3 \\
\hline D & 0.1 & 0.1 & 0.1 & 0.5 & 0.8 & 1.4 & 3.8 & $*$ & $*$ & $*$ \\
\hline c & 0.1 & 0.1 & 0.1 & 0.5 & 0.8 & 1.6 & 6.0 & * & $*$ & $*$ \\
\hline B & 0.1 & 0.2 & 0.2 & 0.5 & 0.7 & 1.5 & 1.4 & * & $*$ & * \\
\hline & & 1 & 2 & 3 & & & 8 & & & 8 \\
\hline
\end{tabular}

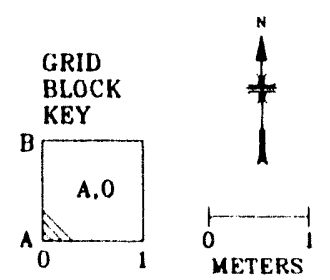

Fig. 11. Gamma exposure rate $(\mathrm{mR} / \mathrm{h}) 1 \mathrm{~m}$ above the floor in each grid block of Building 7819. 
Table 1. Summary of radiation measurements on floor of Building 7819 Decontamination Facility

\begin{tabular}{|c|c|c|c|c|c|c|}
\hline $\begin{array}{l}\text { Location } \\
\text { (Grid ID) }\end{array}$ & $\begin{array}{c}\text { Gamma exposure } \\
\text { rate, center survey } \\
\text { block } 1 \mathrm{~m} \text { above } \\
\text { floor }(\mathrm{mR} / \mathrm{h}) \\
\end{array}$ & $\begin{array}{l}\text { Total alpha } \\
\text { contamination } \\
\left(\mathrm{dpm} / 100 \mathrm{~cm}^{2}\right)\end{array}$ & $\begin{array}{c}\text { Total } \\
\text { beta-gamma } \\
\text { contamination } \\
\left(\mathrm{dpm} / 100 \mathrm{~cm}^{2}\right) \\
\end{array}$ & $\begin{array}{c}\text { Smear } \\
\text { number }\end{array}$ & $\begin{array}{l}\text { Transferable alpha } \\
\text { contamination } \\
\left(\mathrm{dpm} / 100 \mathrm{~cm}^{2}\right)\end{array}$ & $\begin{array}{c}\text { Transferable } \\
\text { beta-gamma } \\
\text { contamination } \\
\left(\mathrm{dpm} / 100 \mathrm{~cm}^{2}\right) \\
\end{array}$ \\
\hline $\mathrm{A}, 0$ & 0.1 & $<100$ & $<1,000$ & 1 & $<20$ & $<200$ \\
\hline $\mathrm{A}, 1$ & 0.2 & $<100$ & 2,700 & 2 & $<20$ & 250 \\
\hline$A, 2$ & 0.2 & $<100$ & 8,000 & 3 & $<20$ & $<200$ \\
\hline$A, 3$ & 0.5 & $<100$ & 18,000 & 4 & $<20$ & 480 \\
\hline$A, 4$ & 0.7 & $<100$ & 55,000 & 5 & $<20$ & $<200$ \\
\hline A, 5 & 1.5 & 140 & 84,000 & 6 & $<20$ & 600 \\
\hline A, 6 & 1.4 & 100 & $a$ & 7 & $<20$ & 210 \\
\hline$A, 7$ & $b$ & $b$ & $b$ & $b$ & $b$ & $b$ \\
\hline A, 8 & $b$ & $b$ & $b$ & $b$ & $b$ & $b$ \\
\hline A,9 & $b$ & $b$ & $b$ & $b$ & $b$ & $b$ \\
\hline $\mathrm{B}, 0$ & 0.1 & $<100$ & 2,300 & 11 & $<20$ & $<200$ \\
\hline $\mathrm{B}, 1$ & 0.1 & $<100$ & 3,000 & 12 & $<20$ & $<200$ \\
\hline $\mathrm{B}, 2$ & 0.1 & $<100$ & 4,900 & 13 & $<20$ & 200 \\
\hline $\mathrm{B}, 3$ & 0.5 & $<100$ & 20,000 & 14 & $<20$ & 310 \\
\hline $\mathrm{B}, 4$ & 0.8 & $<100$ & 24,000 & 15 & $<20$ & 260 \\
\hline $\mathrm{B}, 5$ & 1.6 & 240 & $a$ & 16 & $<20$ & 700 \\
\hline $\mathrm{B}, 6$ & 6.0 & 190 & $a$ & 17 & $<20$ & 1,500 \\
\hline
\end{tabular}


Table 1 (continued)

\begin{tabular}{|c|c|c|c|c|c|c|}
\hline $\begin{array}{l}\text { Location } \\
\text { (Grid ID) }\end{array}$ & $\begin{array}{c}\text { Gamma exposure } \\
\text { rate, center survey } \\
\text { block } 1 \mathrm{~m} \text { above } \\
\text { foor }(\mathrm{mR} / \mathrm{h})\end{array}$ & $\begin{array}{l}\text { Total alpha } \\
\text { contamination } \\
\left(\mathrm{dpm} / 100 \mathrm{~cm}^{2}\right)\end{array}$ & $\begin{array}{c}\text { Total } \\
\text { beta-gamma } \\
\text { contamination } \\
\left(\mathrm{dpm} / 100 \mathrm{~cm}^{2}\right)\end{array}$ & $\begin{array}{c}\text { Smear } \\
\text { number }\end{array}$ & $\begin{array}{l}\text { Transferable alpha } \\
\text { contamination } \\
\left(\mathrm{dpm} / 100 \mathrm{~cm}^{2}\right)\end{array}$ & $\begin{array}{c}\text { Transferable } \\
\text { beta-gamma } \\
\text { contamination } \\
\left(\mathrm{dpm} / 100 \mathrm{~cm}^{2}\right)\end{array}$ \\
\hline $\mathrm{B}, 7$ & $b$ & $b$ & $b$ & $b$ & $b$ & $b$ \\
\hline $\mathrm{B}, 8$ & $b$ & $b$ & $b$ & $b$ & $b$ & $b$ \\
\hline $\mathrm{B}, 9$ & $b$ & $b$ & $b$ & $b$ & $b$ & $b$ \\
\hline $\mathrm{C}, 0$ & 0.1 & $<100$ & 3,300 & 21 & $<20$ & $<200$ \\
\hline $\mathrm{C}, 1$ & 0.1 & $<100$ & 2,100 & 22 & $<20$ & $<200$ \\
\hline $\mathrm{C}, 2$ & 0.1 & $<100$ & 3,300 & 23 & $<20$ & $<200$ \\
\hline $\mathrm{C}, 3$ & 0.5 & $<100$ & 22,000 & 24 & $<20$ & 5,700 \\
\hline$C, 4$ & 0.9 & $<100$ & $a$ & 25 & $<20$ & 280 \\
\hline$C, 5$ & 1.4 & 270 & 20,000 & 26 & $<20$ & 510 \\
\hline$C, 6$ & 3.8 & 200 & 150,000 & 27 & $<20$ & 910 \\
\hline$C, 7$ & $b$ & $b$ & $b$ & $b$ & $b$ & $b$ \\
\hline$C, 8$ & $b$ & $b$ & $b$ & $b$ & $b$ & $b$ \\
\hline $\mathrm{C}, 9$ & $b$ & $b$ & $b$ & $b$ & $b$ & $b$ \\
\hline $\mathrm{D}, 0$ & 0.0 & $<100$ & 12,000 & 31 & $<20$ & $<200$ \\
\hline $\mathrm{D}, 1$ & 0.1 & $<100$ & 4,400 & 32 & $<20$ & $<200$ \\
\hline $\mathrm{D}, 2$ & 0.0 & $<100$ & 1,800 & 33 & $<20$ & $<200$ \\
\hline $\mathrm{D}, 3$ & 0.6 & 130 & 11,000 & 34 & $<20$ & $<200$ \\
\hline
\end{tabular}


Table 1 (continued)

\begin{tabular}{|c|c|c|c|c|c|c|}
\hline $\begin{array}{l}\text { Location } \\
\text { (Grid ID) }\end{array}$ & $\begin{array}{c}\text { Gamma exposure } \\
\text { rate, center survey } \\
\text { block } 1 \mathrm{~m} \text { above } \\
\text { floor }(\mathrm{mR} / \mathrm{h})\end{array}$ & $\begin{array}{c}\text { Total alpha } \\
\text { contamination } \\
\left(\mathrm{dpm} / 100 \mathrm{~cm}^{2}\right)\end{array}$ & $\begin{array}{c}\text { Total } \\
\text { beta-gamma } \\
\text { contamination } \\
\left(\mathrm{dpm} / 100 \mathrm{~cm}^{2}\right) \\
\end{array}$ & $\begin{array}{l}\text { Smear } \\
\text { number }\end{array}$ & $\begin{array}{l}\text { Transferable alpha } \\
\text { contamination } \\
\left(\mathrm{dpm} / 100 \mathrm{~cm}^{2}\right)\end{array}$ & $\begin{array}{c}\text { Transferable } \\
\text { beta-gamma } \\
\text { contamination } \\
\left(\mathrm{dpm} / 100 \mathrm{~cm}^{2}\right)\end{array}$ \\
\hline$D, 4$ & 0.9 & $<100$ & $a$ & 35 & $<20$ & $<200$ \\
\hline $\mathrm{D}, 5$ & 1.5 & 180 & $a$ & 36 & $<20$ & 240 \\
\hline $\mathrm{D}, 6$ & 4.4 & 130 & $a$ & 37 & $<20$ & 530 \\
\hline $\mathrm{D}, 7$ & 10.0 & $<100$ & $a$ & 38 & $<20$ & 560 \\
\hline $\mathrm{D}, 8$ & 5.0 & 270 & $a$ & 39 & $<20$ & 600 \\
\hline $\mathrm{D}, 9$ & 3.3 & 140 & $a$ & 40 & $<20$ & 370 \\
\hline $\mathrm{E}, 0$ & $<0.1$ & $<100$ & 21,000 & 41 & $<20$ & $<200$ \\
\hline$E, 1$ & $<0.1$ & $<100$ & 9,400 & 42 & $<20$ & 360 \\
\hline $\mathrm{E}, 2$ & $<0.1$ & 100 & 21,000 & 43 & $<20$ & 220 \\
\hline$E, 3$ & 0.7 & $<100$ & 8,000 & 44 & 28 & 270 \\
\hline$E, 4$ & 0.8 & $<100$ & $a$ & 45 & $<20$ & 290 \\
\hline E,5 & 1.6 & 200 & $a$ & 46 & $<20$ & 1,600 \\
\hline $\mathrm{E}, 6$ & 2.6 & 200 & $a$ & 47 & $<20$ & 460 \\
\hline $\mathrm{E}, 7$ & 4.3 & 170 & $a$ & 48 & 21 & 550 \\
\hline $\mathrm{E}, 8$ & 3.4 & 470 & $a$ & 49 & $<20$ & 1,500 \\
\hline E,9 & 2.8 & $<100$ & $a$ & 50 & 28 & 4,800 \\
\hline $\mathrm{F}, 0$ & 0.1 & $<100$ & 16,000 & 51 & 28 & $<200$ \\
\hline
\end{tabular}


Table 1 (continued)

\begin{tabular}{|c|c|c|c|c|c|c|}
\hline $\begin{array}{l}\text { Location } \\
\text { (Grid ID) }\end{array}$ & $\begin{array}{c}\text { Gamma exposure } \\
\text { rate, center survey } \\
\text { block } 1 \mathrm{~m} \text { above } \\
\text { floor }(\mathrm{mR} / \mathrm{h})\end{array}$ & $\begin{array}{l}\text { Total alpha } \\
\text { contamination } \\
\left(\mathrm{dpm} / 100 \mathrm{~cm}^{2}\right)\end{array}$ & $\begin{array}{c}\text { Total } \\
\text { beta-gamma } \\
\text { contamination } \\
\left(\mathrm{dpm} / 100 \mathrm{~cm}^{2}\right) \\
\end{array}$ & $\begin{array}{l}\text { Smear } \\
\text { number }\end{array}$ & $\begin{array}{l}\text { Transferable alpha } \\
\text { contamination } \\
\left(\mathrm{dpm} / 100 \mathrm{~cm}^{2}\right)\end{array}$ & $\begin{array}{c}\text { Transferable } \\
\text { beta-gamma } \\
\text { contamination } \\
\left(\mathrm{dpm} / 100 \mathrm{~cm}^{2}\right)\end{array}$ \\
\hline$F, 1$ & 0.1 & $<100$ & 5,400 & 52 & 28 & 260 \\
\hline $\mathrm{F}, 2$ & 0.2 & $<100$ & 3,800 & 53 & $<20$ & 250 \\
\hline$F, 3$ & 0.7 & $<100$ & 10,000 & 54 & 35 & 340 \\
\hline $\mathrm{F}, 4$ & 0.9 & 130 & 4,700 & 55 & 35 & 310 \\
\hline $\mathrm{F}, 5$ & 1.0 & $<100$ & 35,000 & 56 & $<20$ & 2,200 \\
\hline$F, 6$ & 1.5 & $<100$ & 17,000 & 57 & 35 & 230 \\
\hline $\mathrm{F}, 7$ & $b$ & $b$ & $b$ & $b$ & $b$ & $b$ \\
\hline $\mathrm{F}, 8$ & $b$ & $b$ & $b$ & 278 & 56 & 600 \\
\hline $\mathrm{F}, 9$ & $b$ & $b$ & $b$ & $b$ & $b$ & $b$ \\
\hline$G, 0$ & 0.2 & $<100$ & 19,000 & 61 & $<20$ & $<200$ \\
\hline$G, 1$ & 0.2 & $<100$ & 20,000 & 62 & 28 & 700 \\
\hline $\mathrm{G}, 2$ & 0.2 & $<100$ & 11,000 & 63 & $<20$ & 370 \\
\hline G,3 & 0.6 & $<100$ & 20,000 & 64 & $<20$ & 410 \\
\hline$G, 4$ & 0.7 & $<100$ & $a$ & 65 & 28 & 370 \\
\hline G,5 & 0.8 & 200 & $a$ & 66 & 21 & 2,800 \\
\hline G,6 & 0.8 & 100 & 10,000 & 67 & 28 & 380 \\
\hline$G, 7$ & $b$ & $b$ & $b$ & $b$ & $b$ & $b$ \\
\hline
\end{tabular}


Table 1 (continued)

\begin{tabular}{|c|c|c|c|c|c|c|}
\hline $\begin{array}{l}\text { Location } \\
\text { (Grid ID) }\end{array}$ & $\begin{array}{c}\text { Gamma exposure } \\
\text { rate, center survey } \\
\text { block } 1 \mathrm{~m} \text { above } \\
\text { floor }(\mathrm{mR} / \mathrm{h})\end{array}$ & $\begin{array}{l}\text { Total alpha } \\
\text { contamination } \\
\left(\mathrm{dpm} / 100 \mathrm{~cm}^{2}\right)\end{array}$ & $\begin{array}{c}\text { Total } \\
\text { beta-gamma } \\
\text { contamination } \\
\left(\mathrm{dpm} / 100 \mathrm{~cm}^{2}\right) \\
\end{array}$ & $\begin{array}{l}\text { Smear } \\
\text { number }\end{array}$ & $\begin{array}{l}\text { Transferable alpha } \\
\text { contamination } \\
\left(\mathrm{dpm} / 100 \mathrm{~cm}^{2}\right)\end{array}$ & $\begin{array}{c}\text { Transferable } \\
\text { beta-gamma } \\
\text { contamination } \\
\left(\mathrm{dpm} / 100 \mathrm{~cm}^{2}\right) \\
\end{array}$ \\
\hline G,8 & $b$ & $b$ & $b$ & $b$ & $b$ & $b$ \\
\hline G,9 & $b$ & $b$ & $b$ & $b$ & $b$ & $b$ \\
\hline $\mathrm{H}, 0$ & 0.2 & $<100$ & 28,000 & 71 & 35 & 630 \\
\hline $\mathrm{H}, 1$ & 0.2 & $<100$ & 19,000 & 72 & $<20$ & 420 \\
\hline $\mathrm{H}, 2$ & 0.4 & $<100$ & 12,000 & 73 & 28 & $<200$ \\
\hline $\mathrm{H}, 3$ & 0.4 & $<100$ & $<1,000$ & 74 & 42 & 540 \\
\hline $\mathrm{H}, 4$ & 0.5 & $<100$ & $a$ & 75 & 30 & 380 \\
\hline $\mathrm{H}, 5$ & 0.7 & $<100$ & 21,000 & 76 & 70 & 1,500 \\
\hline $\mathrm{H}, 6$ & 1.2 & 120 & 13,000 & 77 & 21 & 280 \\
\hline $\mathrm{H}, 7$ & $b$ & $b$ & $b$ & $b$ & $b$ & $b$ \\
\hline $\mathrm{H}, 8$ & $b$ & $b$ & $b$ & $b$ & $b$ & $b$ \\
\hline $\mathrm{H}, 9$ & $b$ & $b$ & $b$ & $b$ & $b$ & $b$ \\
\hline $\mathrm{I}, 0$ & 0.2 & $<100$ & 10,000 & 81 & 28 & 460 \\
\hline 1,1 & 0.2 & $<100$ & 11,000 & 82 & 28 & 250 \\
\hline $\mathrm{I}, 2$ & 0.3 & $<100$ & 3,100 & 83 & $<20$ & 400 \\
\hline $\mathrm{I}, 3$ & 0.4 & $<100$ & 25,000 & 84 & $<20$ & $<200$ \\
\hline $\mathrm{I}, 4$ & 0.5 & $<100$ & 13,000 & 85 & $<20$ & 300 \\
\hline
\end{tabular}


Table 1 (continued)

\begin{tabular}{|c|c|c|c|c|c|c|}
\hline $\begin{array}{l}\text { Location } \\
\text { (Grid ID) }\end{array}$ & $\begin{array}{c}\text { Gamma exposure } \\
\text { rate, center survey } \\
\text { block } 1 \mathrm{~m} \text { above } \\
\text { floor }(\mathrm{mR} / \mathrm{h})\end{array}$ & $\begin{array}{l}\text { Total alpha } \\
\text { contamination } \\
\left(\mathrm{dpm} / 100 \mathrm{~cm}^{2}\right)\end{array}$ & $\begin{array}{c}\text { Total } \\
\text { beta-gamma } \\
\text { contamination } \\
\left(\mathrm{dpm} / 100 \mathrm{~cm}^{2}\right) \\
\end{array}$ & $\begin{array}{c}\text { Smear } \\
\text { number }\end{array}$ & $\begin{array}{l}\text { Transferable alpha } \\
\text { contamination } \\
\left(\mathrm{dpm} / 100 \mathrm{~cm}^{2}\right)\end{array}$ & $\begin{array}{r}\text { Transferable } \\
\text { beta-gamma } \\
\text { contamination } \\
\left(\mathrm{dpm} / 100 \mathrm{~cm}^{2}\right) \\
\end{array}$ \\
\hline 1,5 & 0.7 & 220 & 44,000 & 86 & $<20$ & 3,400 \\
\hline $\mathrm{I}, 6$ & 1.4 & 120 & 24,000 & 87 & $<20$ & 1,200 \\
\hline $\mathrm{I}, 7$ & $b$ & $b$ & $b$ & $b$ & $b$ & $b$ \\
\hline $\mathrm{I}, 8$ & $b$ & $b$ & $b$ & $b$ & $b$ & $b$ \\
\hline I,9 & $b$ & $b$ & $b$ & $b$ & $b$ & $b$ \\
\hline $\mathrm{J}, 0$ & 0.4 & 120 & 19,000 & 91 & 28 & 750 \\
\hline $\mathrm{J}, 1$ & 0.4 & $<100$ & 6,300 & 92 & 21 & 340 \\
\hline $\mathrm{J}, 2$ & 0.3 & $<100$ & 6,800 & 93 & $<20$ & 470 \\
\hline $\mathrm{J}, 3$ & 0.3 & $<100$ & 6,400 & 94 & $<20$ & 290 \\
\hline $\mathrm{J}, 4$ & 0.4 & 640 & 26,000 & 95 & $<20$ & 620 \\
\hline $\mathrm{J}, 5$ & 0.6 & 240 & 63,000 & 96 & 28 & 8,100 \\
\hline $\mathrm{J}, 6$ & 0.9 & 400 & 59,000 & 97 & $<20$ & 1,900 \\
\hline $\mathrm{J}, 7$ & $b$ & $b$ & $b$ & $b$ & $b$ & $b$ \\
\hline $\mathrm{J}, 8$ & $b$ & $b$ & $b$ & $b$ & $b$ & $b$ \\
\hline $\mathrm{J}, 9$ & $b$ & $b$ & $b$ & $b$ & $b$ & $b$ \\
\hline $\mathrm{K}, 0$ & 0.5 & $<100$ & 13,000 & 101 & 28 & 1,400 \\
\hline $\mathrm{K}, 1$ & 0.6 & $b$ & $b$ & $b$ & $b$ & $b$ \\
\hline
\end{tabular}


Table 1 (continued)

\begin{tabular}{|c|c|c|c|c|c|c|}
\hline $\begin{array}{l}\text { Location } \\
\text { (Grid ID) }\end{array}$ & $\begin{array}{l}\text { Gamma exposure } \\
\text { rate, center survey } \\
\text { block } 1 \mathrm{~m} \text { above } \\
\text { floor }(\mathrm{mR} / \mathrm{h})\end{array}$ & $\begin{array}{c}\text { Total alpha } \\
\text { contamination } \\
\left(\mathrm{dpm} / 100 \mathrm{~cm}^{2}\right)\end{array}$ & $\begin{array}{c}\text { Total } \\
\text { beta-gamma } \\
\text { contamination } \\
\left(\mathrm{dpm} / 100 \mathrm{~cm}^{2}\right)\end{array}$ & $\begin{array}{c}\text { Smear } \\
\text { number }\end{array}$ & $\begin{array}{l}\text { Transferable alpha } \\
\text { contamination } \\
\left(\mathrm{dpm} / 100 \mathrm{~cm}^{2}\right)\end{array}$ & $\begin{array}{c}\text { Transferable } \\
\text { beta-gamma } \\
\text { contamination } \\
\left(\mathrm{dpm} / 100 \mathrm{~cm}^{2}\right) \\
\end{array}$ \\
\hline $\mathrm{K}, 2$ & 0.4 & 120 & 4,200 & 103 & $<20$ & 480 \\
\hline $\mathrm{K}, 3$ & 0.3 & $<100$ & 5,100 & 104 & 28 & 440 \\
\hline $\mathrm{K}, 4$ & 0.4 & 300 & 6,700 & 105 & 49 & 4,500 \\
\hline $\mathrm{K}, 5$ & 0.5 & 250 & 190,000 & 106 & $<20$ & 10,000 \\
\hline $\mathrm{K}, 6$ & 1.5 & 13,000 & 520,000 & 107 & 230 & 31,000 \\
\hline $\mathrm{K}, 7$ & 20.0 & 900 & 99,000 & 108 & 98 & 50,000 \\
\hline $\mathrm{K}, 7$ & $b$ & $b$ & $b$ & 279 & 100 & 6,800 \\
\hline $\mathrm{K}, 8$ & 1.3 & 220 & 11,000 & 109 & $<20$ & 20,000 \\
\hline $\mathrm{K}, 9$ & 0.7 & 230 & $a$ & 110 & 35 & 3,500 \\
\hline $\mathrm{L}, 0$ & 1.1 & $<100$ & 26,000 & 111 & $<20$ & 280 \\
\hline $\mathrm{L}, 1$ & 0.4 & 120 & $a$ & 112 & $<20$ & 250 \\
\hline $\mathrm{L}, 2$ & 0.3 & $<100$ & 6,400 & 113 & $<20$ & 1,000 \\
\hline $\mathrm{L}, 3$ & 0.3 & 120 & 4,700 & 114 & $<20$ & 480 \\
\hline $\mathrm{L}, 4$ & 0.5 & 170 & 5,000 & 115 & $<20$ & 610 \\
\hline$L, 5$ & 0.5 & 450 & 49,000 & 116 & $<20$ & 1,300 \\
\hline L,6 & 0.8 & 1,300 & 150,000 & 117 & $c$ & $c$ \\
\hline $\mathrm{L}, 7$ & 1.4 & 950 & 190,000 & 118 & $c$ & $c$ \\
\hline
\end{tabular}


Table 1 (continued)

\begin{tabular}{|c|c|c|c|c|c|c|}
\hline $\begin{array}{l}\text { Location } \\
\text { (Grid ID) }\end{array}$ & $\begin{array}{c}\text { Gamma exposure } \\
\text { rate, center survey } \\
\text { block } 1 \mathrm{~m} \text { above } \\
\text { floor }(\mathrm{mR} / \mathrm{h})\end{array}$ & $\begin{array}{c}\text { Total alpha } \\
\text { contamination } \\
\left(\mathrm{dpm} / 100 \mathrm{~cm}^{2}\right)\end{array}$ & $\begin{array}{c}\text { Total } \\
\text { beta-gamma } \\
\text { contamination } \\
\left(\mathrm{dpm} / 100 \mathrm{~cm}^{2}\right) \\
\end{array}$ & $\begin{array}{c}\text { Smear } \\
\text { number }\end{array}$ & $\begin{array}{l}\text { Transferable alpha } \\
\text { contamination } \\
\left(\mathrm{dpm} / 100 \mathrm{~cm}^{2}\right)\end{array}$ & $\begin{array}{c}\text { Transferable } \\
\text { beta-gamma } \\
\text { contamination } \\
\left(\mathrm{dpm} / 100 \mathrm{~cm}^{2}\right)\end{array}$ \\
\hline $\mathrm{L}, 8$ & 1.7 & 260 & 96,000 & 119 & $c$ & $c$ \\
\hline L,9 & 0.6 & 300 & $a$ & 120 & $c$ & $c$ \\
\hline $\mathrm{M}, 0$ & 4.8 & 180 & $a$ & 121 & $<20$ & 290 \\
\hline M,1 & 1.5 & $<100$ & $a$ & 122 & 21 & 560 \\
\hline $\mathrm{M}, 2$ & 1.0 & $<100$ & 1,200 & 123 & 21 & 380 \\
\hline $\mathrm{M}, 3$ & 0.5 & $<100$ & 2,700 & 124 & $<20$ & 440 \\
\hline $\mathrm{M}, 4$ & 0.5 & 170 & 23,000 & 125 & $<20$ & 800 \\
\hline M,5 & 0.4 & 400 & 41,000 & 126 & 35 & 2,800 \\
\hline M,6 & 0.6 & 140 & 100,000 & 127 & $<20$ & 2,200 \\
\hline $\mathrm{M}, 7$ & 1.2 & 190 & 79,000 & 128 & 28 & 2,600 \\
\hline $\mathbf{M}, \mathbf{8}$ & 2.6 & 310 & 22,000 & 129 & $<20$ & 2,600 \\
\hline M,9 & 1.2 & 190 & 25,000 & 130 & 21 & 1,600 \\
\hline $\mathrm{N}, 0$ & 1.7 & $<100$ & 3,000 & 131 & $<20$ & 580 \\
\hline $\mathrm{N}, 1$ & 0.8 & $<100$ & 5,600 & 132 & $<20$ & 390 \\
\hline $\mathrm{N}, 2$ & 0.4 & $<100$ & 3,900 & 133 & $<20$ & 310 \\
\hline $\mathrm{N}, 3$ & 0.3 & 140 & 10,000 & 134 & $<20$ & $<200$ \\
\hline $\mathbf{N}, 4$ & 0.2 & 180 & $a$ & 135 & $<20$ & 990 \\
\hline
\end{tabular}


Table 1 (continued)

\begin{tabular}{|c|c|c|c|c|c|c|}
\hline $\begin{array}{l}\text { Location } \\
\text { (Grid ID) }\end{array}$ & $\begin{array}{l}\text { Gamma exposure } \\
\text { rate, center survey } \\
\text { block } 1 \mathrm{~m} \text { above } \\
\text { floor }(\mathrm{mR} / \mathrm{h})\end{array}$ & $\begin{array}{c}\text { Total alpha } \\
\text { contamination } \\
\left(\mathrm{dpm} / 100 \mathrm{~cm}^{2}\right)\end{array}$ & $\begin{array}{c}\text { Total } \\
\text { beta-gamma } \\
\text { contamination } \\
\left(\mathrm{dpm} / 100 \mathrm{~cm}^{2}\right)\end{array}$ & $\begin{array}{c}\text { Smear } \\
\text { number }\end{array}$ & $\begin{array}{l}\text { Transferable alpha } \\
\text { contamination } \\
\left(\mathrm{dpm} / 100 \mathrm{~cm}^{2}\right)\end{array}$ & $\begin{array}{c}\text { Transferable } \\
\text { beta-gamma } \\
\text { contamination } \\
\left(\mathrm{dpm} / 100 \mathrm{~cm}^{2}\right)\end{array}$ \\
\hline $\mathrm{N}, 5$ & 0.2 & 280 & 26,000 & 136 & $<20$ & 270 \\
\hline $\mathrm{N}, 6$ & 0.6 & 380 & $a$ & 137 & $<20$ & 330 \\
\hline $\mathrm{N}, 7$ & 0.9 & 510 & 280,000 & 138 & $<20$ & 610 \\
\hline $\mathrm{N}, 8$ & 1.7 & 170 & $a$ & 139 & 21 & 4,600 \\
\hline $\mathrm{N}, 9$ & 1.0 & $<100$ & 14,000 & 140 & $<20$ & 350 \\
\hline 0,0 & 0.3 & 100 & 11,000 & 141 & $<20$ & 310 \\
\hline 0,1 & 0.3 & $<100$ & 51,000 & 142 & $<20$ & 330 \\
\hline 0,2 & 0.3 & 190 & 48,000 & 143 & $<20$ & $<200$ \\
\hline 0,3 & 0.3 & 180 & 16,000 & 144 & $<20$ & $<200$ \\
\hline 0,4 & $b$ & $b$ & $b$ & $b$ & $b$ & $b$ \\
\hline 0,5 & $b$ & $b$ & $b$ & $b$ & $b$ & $b$ \\
\hline 0,6 & 0.1 & 330 & 370,000 & 147 & 21 & 340 \\
\hline 0,7 & 0.5 & 240 & 31,000 & 148 & 21 & 1,300 \\
\hline 0,8 & 0.9 & 230 & 13,000 & 149 & $<20$ & 900 \\
\hline 0,9 & 0.7 & $b$ & $b$ & 150 & $b$ & $b$ \\
\hline
\end{tabular}

${ }_{b}^{a}$ Radioactive shine from surrounding objects prevented determination of total beta-gamma contamination.

b Inaccessible.

${ }^{c}$ Not removed from envelope. Selected for further analysis. 
ORNL Photo 5959-93

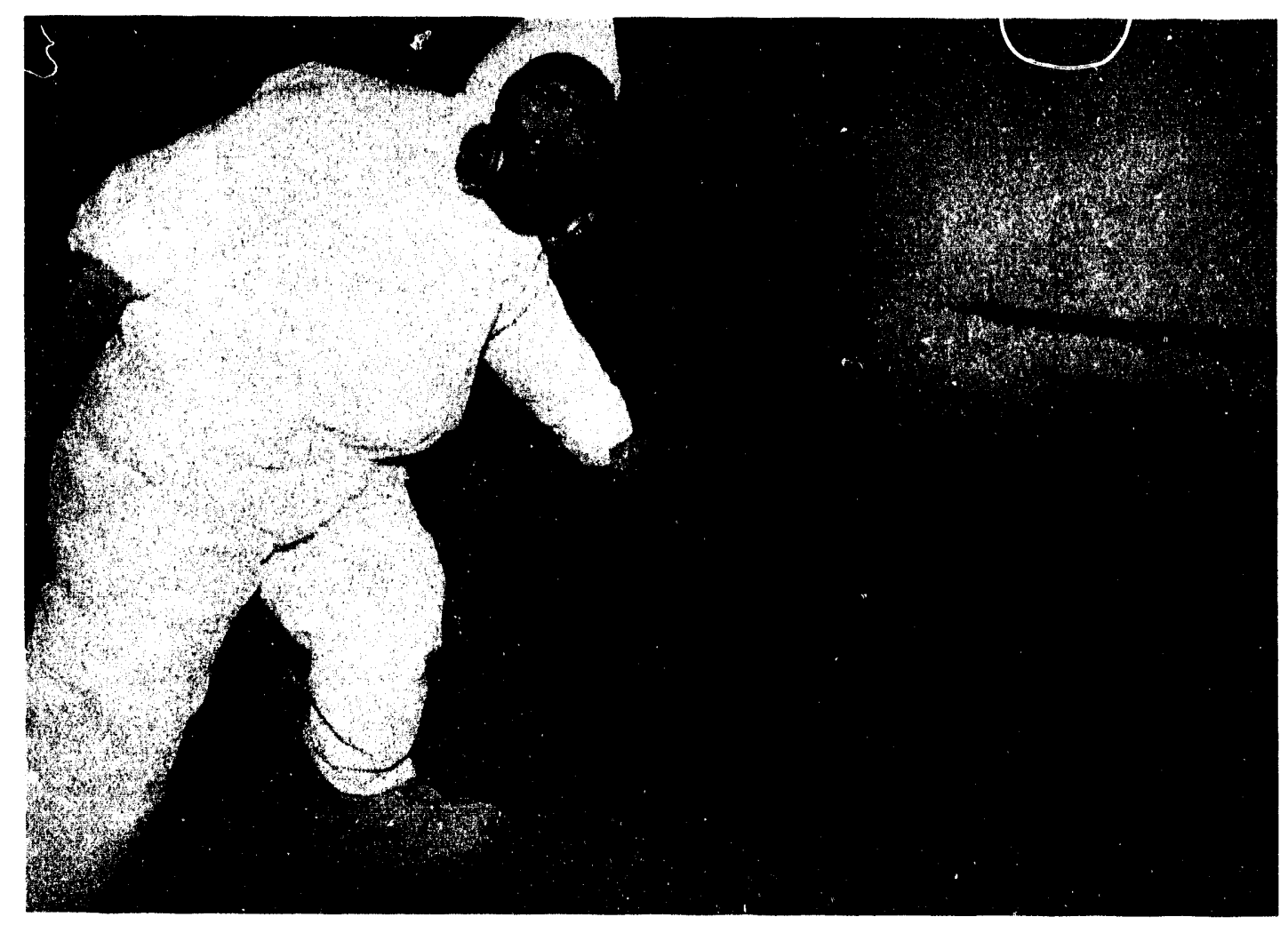

Fig. 12. View of highly contaminated pump in southeastem end of Building 7819 (July 1993). 
ORNL Photo 5970-93

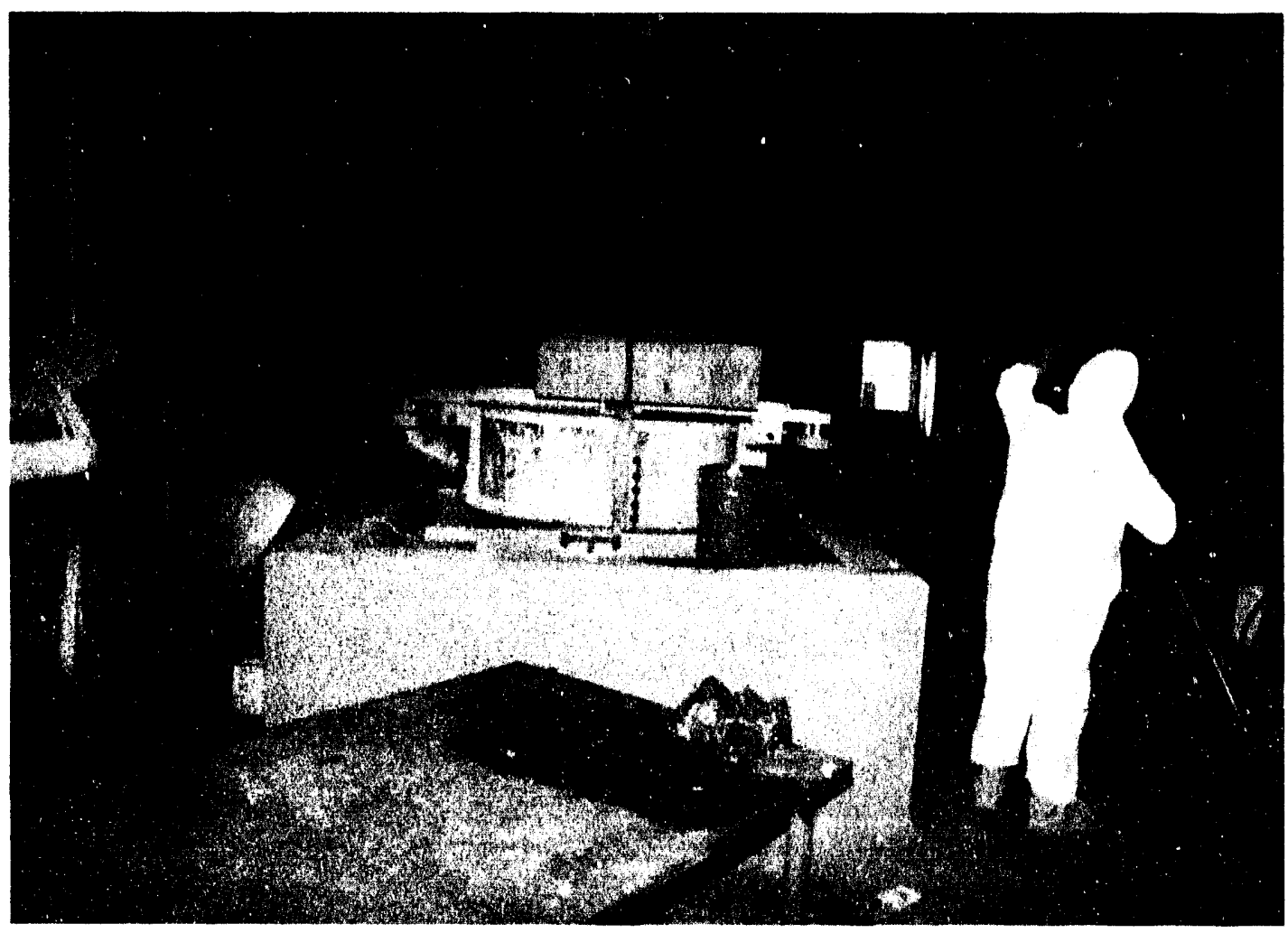

Fig. 13. View of contaminated metal table in northeastern side of Building 7819 (July 1993). 
ORNL Photo 5963-93

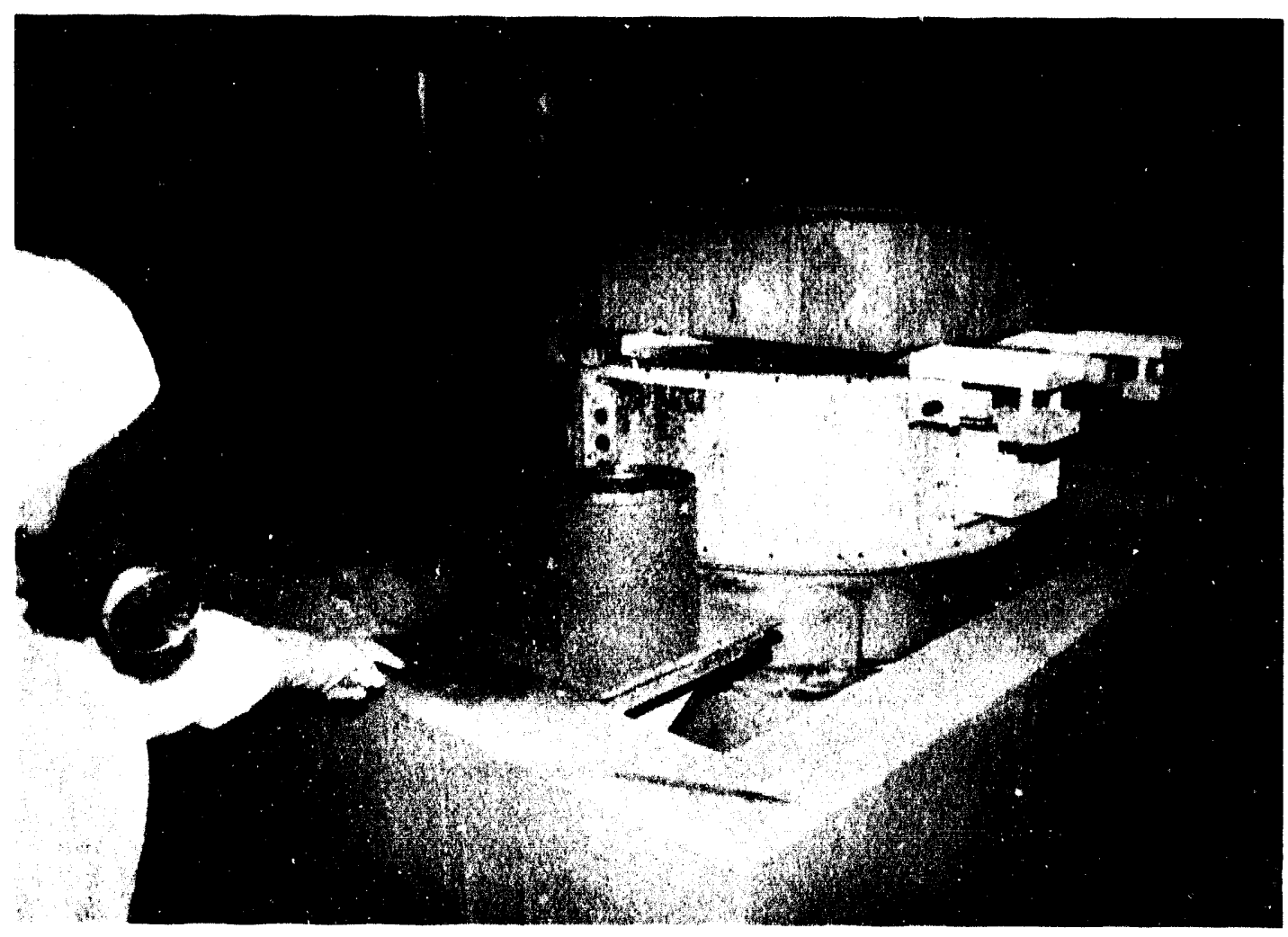

Fig. 14. View of grossly contaminated ledge above sink in northeastern end of Building 7819 (July 1993). 


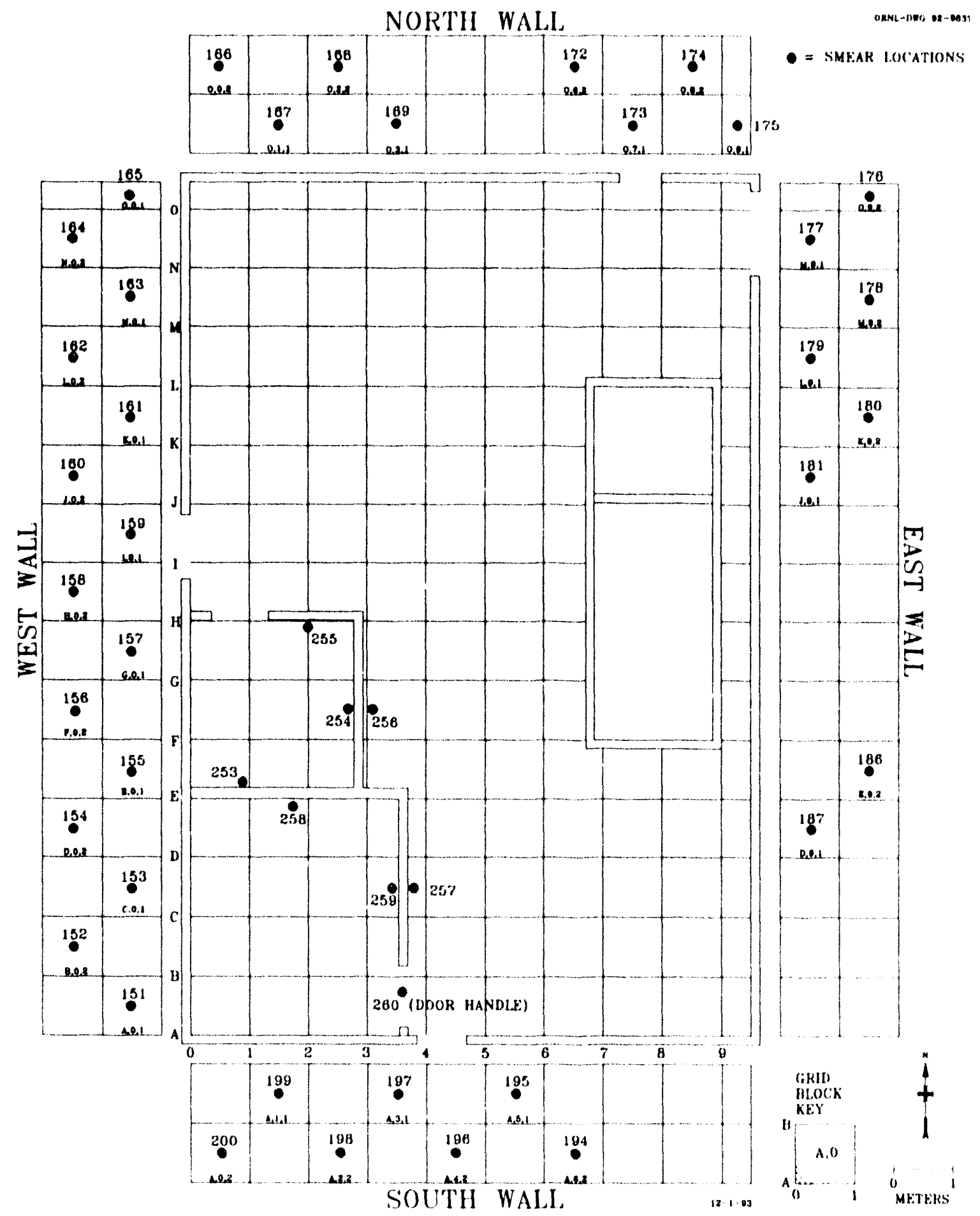

Fig. 15. Sampling locations for transferable alpha and beta-gamma contamination on walls in Building 7819. 


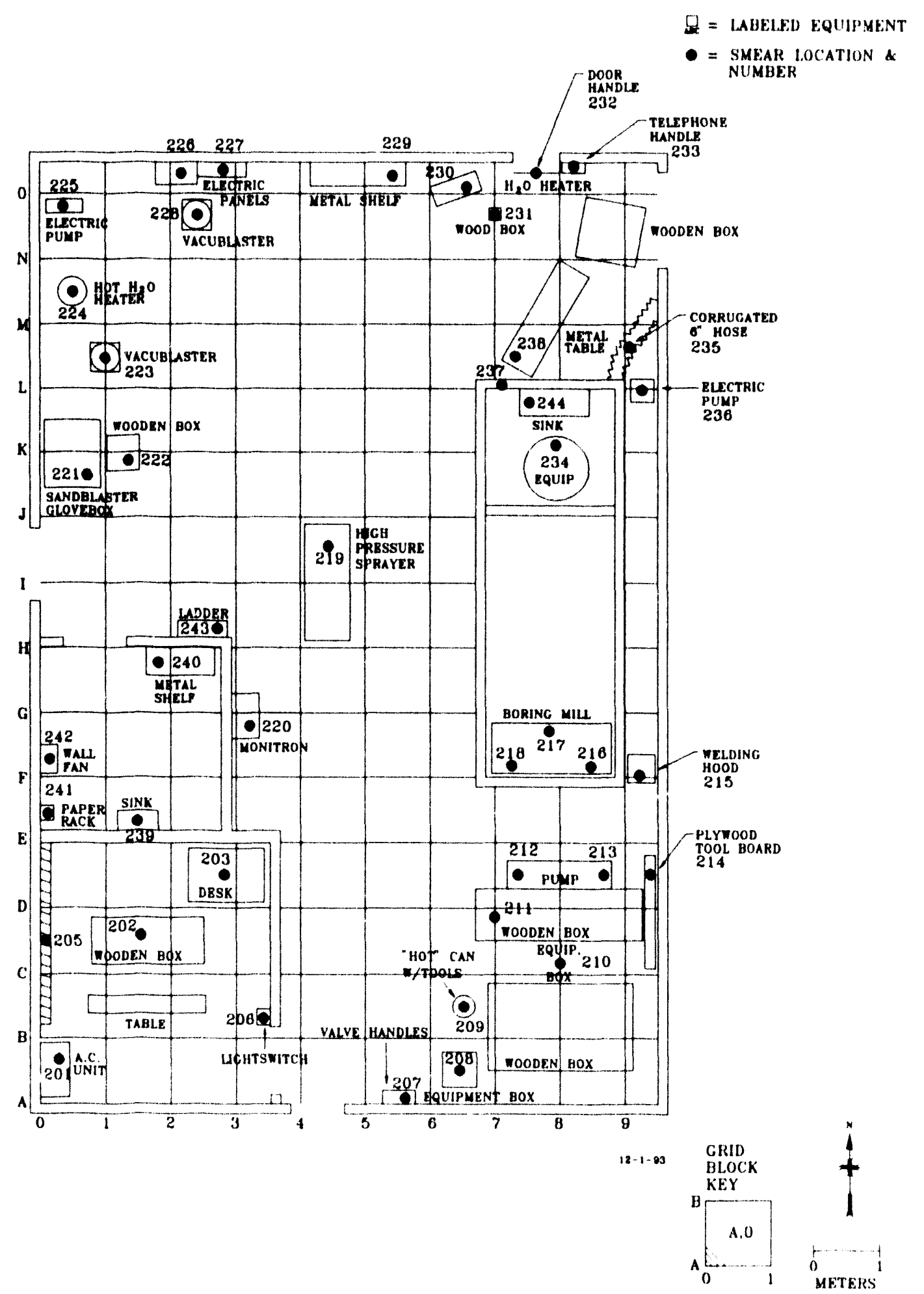

Fig. 16. Sampling locations for transferable alpha and beta-gamma contamination on equipment in Building 7819. 
Figures 17 and 18, respectively, indicate the locations of samples taken from drains and overhead surfaces. Transferable alpha and beta-gamma contamination at smear locations on walls, equipment, drains, and overhead surfaces is shown in Tables 2-5, respectively, in disintegrations $/$ minute $/ 100 \mathrm{~cm}^{2}$.

Table 6 shows results of radionuclide analysis of a subset of 11 samples taken from areas of elevated radioactivity in Building 7819 and sent to ORNL Analytical Chemistry Division for analysis. Sample results show the primary beta-emitters to be ${ }^{90} \mathrm{Sr}$ and ${ }^{137} \mathrm{Cs}$ with trace amounts of ${ }^{60} \mathrm{Co}$. Analysis of smear samples to identify alpha-emitting radionuclides was inconclusive, because insufficient amounts of transferable alpha activity were picked up on the smear samples during collection.

Air sample results showed net activity levels less than the Minimum Detectable Activity (MDA) of $22 \mathrm{dpm}$ for beta and less than the MDA of $1.5 \mathrm{dpm}$ for alpha. When the MDA was used to calculate results, gross beta contamination was less than $0.2 \%$ of the Derived Air Concentration (DAC) for ${ }^{90} \mathrm{Sr}$, and gross alpha contamination was less than $9 \%$ of the DAC for ${ }^{239} \mathrm{Pu}$. 
ORNL-DWG 93-16429

$$
\begin{aligned}
(B)= & \text { FLOOR DRAIN \& } \\
& \text { SMEAR LOCATION } \\
(\text { OP. })= & \text { OPEN DRAIN } \\
(\text { CL. })= & \text { CLOSED DRAIN }
\end{aligned}
$$

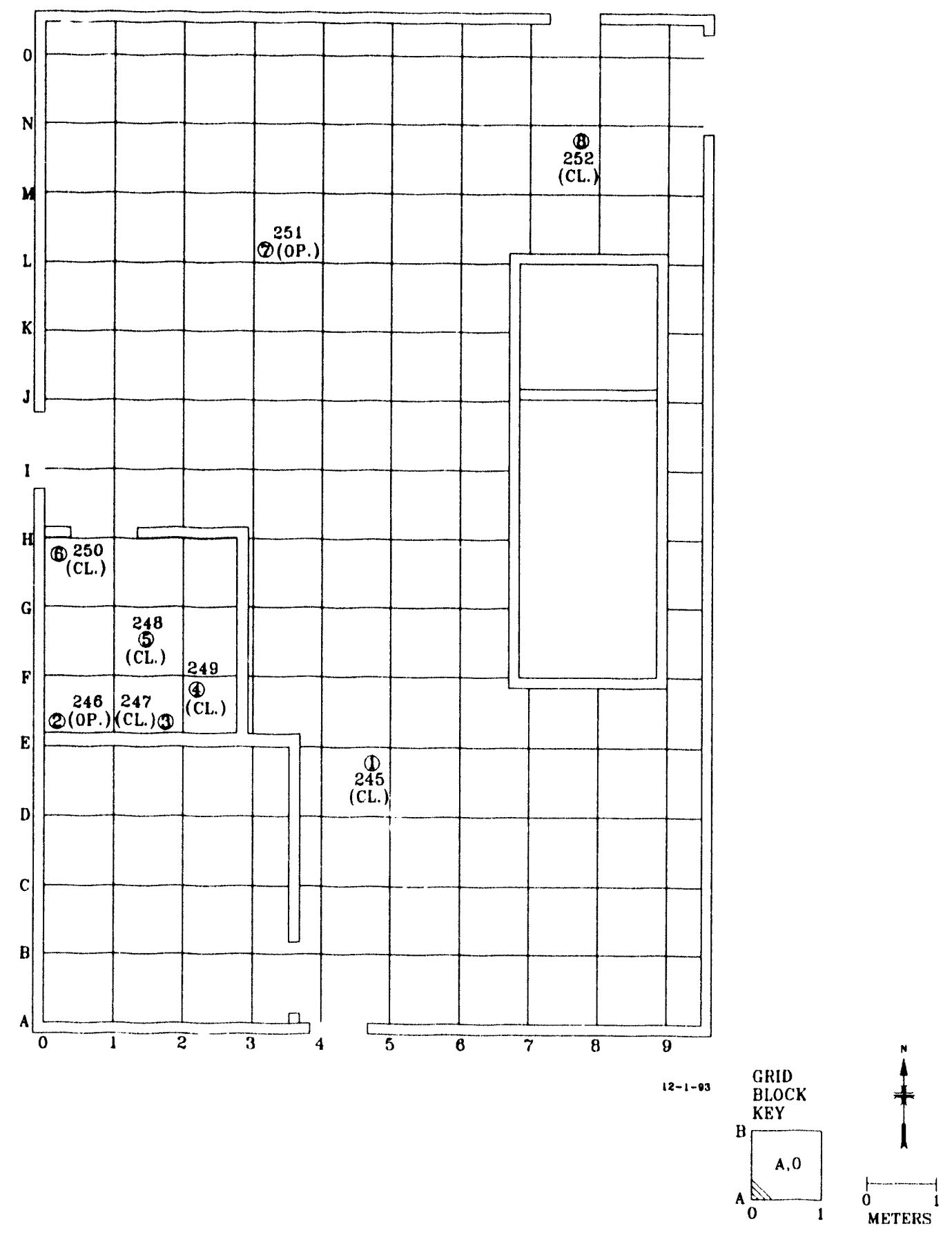

Fig. 17. Sampling locations for transferable alpha and beta-gamma contamination on drains in Building 7819. 
ORNL-DNG 93-10420
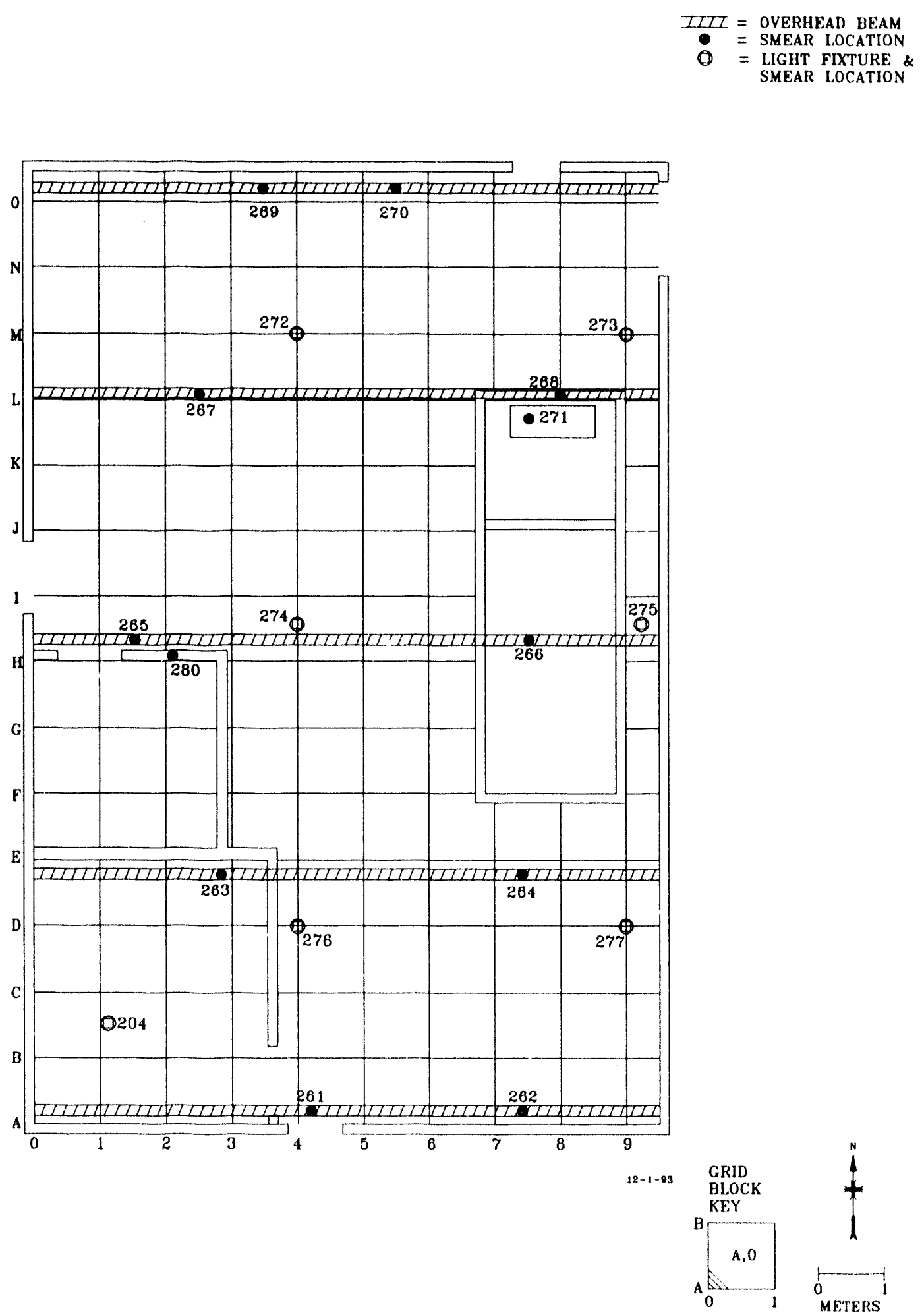

Fig. 18. Sampling locations for transferable alpha and beta-gamma contamination on overhead surfaces in Building 7819. 
Table 2. Summary of radiation measurements on interior and exterior walls of Building 7819 Decontamination Facility

\begin{tabular}{|c|c|c|c|}
\hline $\begin{array}{l}\text { Location } \\
\text { (Grid ID) }\end{array}$ & Smear number & $\begin{array}{c}\text { Transferable alpha } \\
\text { contamination } \\
\left(\mathrm{dpm} / 100 \mathrm{~cm}^{2}\right) \\
\end{array}$ & $\begin{array}{l}\text { Transferable beta- } \\
\text { gamma contamination } \\
\left(\mathrm{dpm} / 100 \mathrm{~cm}^{2}\right)\end{array}$ \\
\hline \multicolumn{4}{|c|}{ Exterior Walls ${ }^{a}$} \\
\hline$A, 0,1$ & 151 & $<20$ & $<200$ \\
\hline $\mathrm{B}, 0,2$ & 152 & $<20$ & $<200$ \\
\hline $\mathrm{C}, 0,1$ & 153 & $<20$ & $<200$ \\
\hline $\mathrm{D}, 0,2$ & 154 & 21 & $<200$ \\
\hline $\mathrm{E}, 0,1$ & 155 & $<20$ & $<200$ \\
\hline $\mathrm{F}, 0,2$ & 156 & $<20$ & $<200$ \\
\hline$G, 0,1$ & 157 & $<20$ & $<200$ \\
\hline $\mathrm{H}, 0,2$ & 158 & $<20$ & 200 \\
\hline $\mathrm{I}, 0,1$ & 159 & $<20$ & $<200$ \\
\hline $\mathrm{J}, 0,2$ & 160 & 21 & 540 \\
\hline $\mathrm{K}, 0,1$ & 161 & $<20$ & $<200$ \\
\hline $\mathrm{L}, 0,2$ & 162 & 21 & 270 \\
\hline $\mathrm{M}, 0,1$ & 163 & $<20$ & $<200$ \\
\hline $\mathrm{N}, 0,2$ & 164 & $<20$ & $<200$ \\
\hline $0,0,1$ & 165 & $<20$ & $<200$ \\
\hline $0,0,2$ & 166 & $<20$ & $<200$ \\
\hline $0,1,1$ & 167 & $<20$ & $<200$ \\
\hline $0,2,2$ & 168 & $<20$ & $<200$ \\
\hline $0,3,1$ & 169 & $<20$ & $<200$ \\
\hline $0,4,2$ & $b$ & $b$ & $b$ \\
\hline $0,5,1$ & $b$ & $b$ & $b$ \\
\hline $0,6,2$ & 172 & $<20$ & $<200$ \\
\hline $0,7,1$ & 173 & 28 & 700 \\
\hline $0,8,2$ & 174 & $<20$ & $<200$ \\
\hline $0,9,1$ & 175 & $<20$ & $<200$ \\
\hline $0,9,2$ & 176 & $<20$ & $<200$ \\
\hline $\mathrm{N}, 9,1$ & 177 & $<20$ & $<200$ \\
\hline
\end{tabular}


Table 2 (continued)

\begin{tabular}{|c|c|c|c|}
\hline $\begin{array}{l}\text { Location } \\
\text { (Grid ID) }\end{array}$ & Smear number & $\begin{array}{c}\text { Transferable alpha } \\
\text { contamination } \\
\left(\mathrm{dpm} / 100 \mathrm{~cm}^{2}\right)\end{array}$ & $\begin{array}{l}\text { Transferable beta- } \\
\text { gamma contamination } \\
\left(\mathrm{dpm} / 100 \mathrm{~cm}^{2}\right)\end{array}$ \\
\hline $\mathrm{M}, 9,2$ & 178 & $<20$ & $<200$ \\
\hline L,9,1 & 179 & $<20$ & $<200$ \\
\hline K,9,2 & 180 & $<20$ & $<200$ \\
\hline$J, 9,1$ & 181 & $<20$ & $<200$ \\
\hline $1,9,2$ & $b$ & $b$ & $b$ \\
\hline $\mathrm{H}, 9,1$ & $b$ & $b$ & $b$ \\
\hline$G, 9,2$ & $b$ & $b$ & $b$ \\
\hline$F, 9,1$ & $b$ & $b$ & $b$ \\
\hline $\mathrm{E}, 9,2$ & 186 & $<20$ & $<200$ \\
\hline $\mathrm{D}, 9,1$ & 187 & $<20$ & $<200$ \\
\hline $\mathrm{C}, 9,2$ & $b$ & $b$ & $b$ \\
\hline $\mathrm{B}, 9,1$ & $b$ & $b$ & $b$ \\
\hline A, 9,2 & $b$ & $b$ & $b$ \\
\hline$A, 9,1$ & $b$ & $b$ & $b$ \\
\hline A, 8,2 & $b$ & $b$ & $b$ \\
\hline A, 7,1 & $b$ & $b$ & $b$ \\
\hline A, 6,2 & 194 & $<20$ & $<200$ \\
\hline A, 5,1 & 195 & 21 & $<200$ \\
\hline$A, 4,2$ & 196 & $<20$ & $<200$ \\
\hline$A, 3,1$ & 197 & $<20$ & $<200$ \\
\hline A, 2,2 & 198 & $<20$ & $<200$ \\
\hline A, 1,1 & 199 & $<20$ & $<200$ \\
\hline$A, 0,2$ & 200 & $<20$ & $<200$ \\
\hline
\end{tabular}


Table 2 (continued)

\begin{tabular}{cccc}
\hline $\begin{array}{c}\text { Location } \\
\text { (Grid ID) }\end{array}$ & Smear number & $\begin{array}{c}\text { Transferable alpha } \\
\text { contamination } \\
\left(\mathrm{dpm} / 100 \mathrm{~cm}^{2}\right)\end{array}$ & $\begin{array}{c}\text { Transferable beta- } \\
\text { gamma contamination } \\
\left(\mathrm{dpm} / 100 \mathrm{~cm}^{2}\right)\end{array}$ \\
\hline E,0 & \multicolumn{2}{c}{ Interior Walls $^{\mathrm{c}}$} & $<200$ \\
F,2 & 253 & $<20$ & $<200$ \\
G,2 & 254 & $<20$ & $<200$ \\
F,3 & 255 & $<20$ & $<200$ \\
C,3 & 256 & $<20$ & $<200$ \\
D,1 & 257 & $<20$ & $<200$ \\
C,3 & 258 & $<20$ & $<200$ \\
A,3 & 259 & $<20$ & $<200$ \\
\hline
\end{tabular}

a Smear samples from exterior walls were taken at $0.5 \mathrm{~m}$ and $1.5 \mathrm{~m}$ above the ground in alternate grids.

${ }^{b}$ Inaccessible.

c Smear samples from interior walls were taken $1 \mathrm{~m}$ above the floor. 
Table 3. Summary of radiation measurements on equipment in Building 7819 Decontamination Facility

\begin{tabular}{|c|c|c|c|}
\hline $\begin{array}{l}\text { Location } \\
\text { (Grid ID) }\end{array}$ & Smear number & $\begin{array}{c}\text { Transferable alpha } \\
\text { contamination } \\
\left(\mathrm{dpm} / 100 \mathrm{~cm}^{2}\right)\end{array}$ & $\begin{array}{l}\text { Transferable } \\
\text { beta-gamma } \\
\text { contamination } \\
\left(\mathrm{dpm} / 100 \mathrm{~cm}^{2}\right)\end{array}$ \\
\hline$A, 0$ & 201 & $<20$ & $<200$ \\
\hline $\mathrm{C}, 1$ & 202 & 28 & 260 \\
\hline $\mathrm{D}, 2$ & 203 & $<20$ & $<200$ \\
\hline $\mathrm{C}, 0$ & 205 & $<20$ & $<200$ \\
\hline $\mathrm{B}, 3$ & 206 & $<20$ & $<200$ \\
\hline A,5 & 207 & 21 & $<200$ \\
\hline A, 6 & 208 & $<20$ & $<200$ \\
\hline $\mathrm{B}, 6$ & 209 & 21 & $<200$ \\
\hline $\mathrm{C}, 8$ & 210 & $<20$ & $<200$ \\
\hline $\mathrm{C}, 7$ & 211 & $<20$ & $<200$ \\
\hline $\mathrm{D}, 7$ & 212 & $<20$ & 2,000 \\
\hline $\mathrm{D}, 8$ & 213 & 56 & 59,000 \\
\hline $\mathrm{D}, 9$ & 214 & 290 & 6,800 \\
\hline$F, 9$ & 215 & $<20$ & 660 \\
\hline $\mathrm{F}, 8$ & 216 & $<20$ & 290 \\
\hline $\mathrm{F}, 7$ & 217 & $<20$ & $<200$ \\
\hline $\mathrm{F}, 7$ & 218 & 91 & 530 \\
\hline$I, 4$ & 219 & $<20$ & $<200$ \\
\hline $\mathrm{F}, 3$ & 220 & $<20$ & $<200$ \\
\hline $\mathrm{J}, 0$ & 221 & $<20$ & 1,200 \\
\hline $\mathrm{J}, 1$ & 222 & 21 & $<200$ \\
\hline $\mathrm{L}, 1$ & 223 & $<20$ & $<200$ \\
\hline $\mathrm{M}, 0$ & 224 & $<20$ & $<200$ \\
\hline $\mathrm{N}, 0$ & 225 & $<20$ & 320 \\
\hline 0,2 & 226 & $<20$ & $<200$ \\
\hline 0,2 & 227 & $<20$ & $<200$ \\
\hline $\mathrm{N}, 2$ & 228 & 21 & 600 \\
\hline
\end{tabular}


Table 3 (continued)

\begin{tabular}{cccc}
\hline $\begin{array}{c}\text { Location } \\
\text { (Grid ID) }\end{array}$ & Smear number & $\begin{array}{c}\text { Transferable alpha } \\
\text { contamination } \\
\left(\mathrm{dpm} / 100 \mathrm{~cm}^{2}\right)\end{array}$ & $\begin{array}{c}\text { Transferable } \\
\text { beta-gamma } \\
\text { contamination } \\
\left.\text { (dpm } / 100 \mathrm{~cm}^{2}\right)\end{array}$ \\
\hline O,5 & 229 & 21 & $<200$ \\
O,6 & 230 & $<20$ & $<200$ \\
N,7 & 231 & $<20$ & 730 \\
O,7 & 232 & 21 & 580 \\
O,8 & 233 & 42 & 1,300 \\
K,7 & 234 & $<20$ & 280 \\
L,9 & 235 & 49 & 850 \\
L,9 & 236 & $<20$ & 300 \\
L,7 & 237 & 440 & 17,000 \\
L,7 & 238 & 270 & 58,000 \\
E,1 & 239 & $<20$ & 430 \\
G,1 & 240 & $<20$ & $<200$ \\
E,0 & 241 & $<20$ & $<200$ \\
F,0 & 242 & $<20$ & $<200$ \\
H,2 & 243 & 28 & $<200$ \\
K,7 & 244 & 56 & \\
\hline
\end{tabular}


Table 4. Summary of radiation measurements on drains in Building 7819 Liscontamination Facility

\begin{tabular}{cccc}
\hline $\begin{array}{c}\text { Location } \\
\text { (Grid ID) }\end{array}$ & Smear number & $\begin{array}{c}\text { Transferable alpha } \\
\text { contamination } \\
\left(\mathrm{dpm} / 100 \mathrm{~cm}^{2}\right)\end{array}$ & $\begin{array}{c}\text { Transferable } \\
\text { beta-gamma } \\
\text { contamination } \\
\left(\mathrm{dpm} / 100 \mathrm{~cm}^{2}\right)\end{array}$ \\
\hline D,4 & 245 & $<20$ & 820 \\
$\mathrm{E}, 0$ & 246 & $<20$ & 610 \\
$\mathrm{E}, 1$ & 247 & $<20$ & $<200$ \\
F,1 & 248 & $<20$ & 260 \\
F,2 & 249 & $<20$ & $<200$ \\
$\mathrm{G}, 0$ & 250 & $<20$ & $<200$ \\
$\mathrm{~L}, 3$ & 251 & $<20$ & 370 \\
M,7 & 252 & $<20$ & 900 \\
\hline
\end{tabular}


Table 5. Summary of overhead radiation measurements in Building 7819 Decontamination Facility

\begin{tabular}{|c|c|c|}
\hline Smear number & $\begin{array}{l}\text { Transferable alpha } \\
\text { contamination } \\
\left(\mathrm{dpm} / 100 \mathrm{~cm}^{2}\right)\end{array}$ & $\begin{array}{c}\text { Transferable beta-gamma } \\
\text { contamination } \\
\left(\mathrm{dpm} / 100 \mathrm{~cm}^{2}\right)\end{array}$ \\
\hline 204 & $<20$ & 260 \\
\hline 261 & $<20$ & $<200$ \\
\hline 262 & $<20$ & $<200$ \\
\hline 263 & $<20$ & $<200$ \\
\hline 264 & $<20$ & $<200$ \\
\hline 265 & $<20$ & $<200$ \\
\hline 266 & $<20$ & $<200$ \\
\hline 267 & $<20$ & $<200$ \\
\hline 268 & $<20$ & $<200$ \\
\hline 269 & $<20$ & $<200$ \\
\hline 270 & $<20$ & $<200$ \\
\hline 271 & $<20$ & $<200$ \\
\hline 272 & $<20$ & $<200$ \\
\hline 273 & $<20$ & $<200$ \\
\hline 274 & $<20$ & $<200$ \\
\hline 275 & $<20$ & 350 \\
\hline 276 & $<20$ & 520 \\
\hline 277 & $<20$ & 380 \\
\hline 280 & $<20$ & $<200$ \\
\hline
\end{tabular}


Table 6 . Activity levels of gross alpha, gross beta, ${ }^{95},{ }^{60} \mathrm{Co}$, and ${ }^{137} \mathrm{Cs}$

in smear samples from Building 7819 Decontamination Facility

\begin{tabular}{|c|c|c|c|c|c|}
\hline \multirow{2}{*}{ Smear number } & \multicolumn{5}{|c|}{ Activity $\left(\mathrm{dpm} / 100 \mathrm{~cm}^{2}\right)$} \\
\hline & Gross alpha ${ }^{a}$ & Gross beta & ${ }^{90} \mathrm{Sr}$ & ${ }^{60} \mathrm{Co}$ & ${ }^{137} \mathrm{Cs}$ \\
\hline 96 & $<600$ & 5,580 & 2,640 & $b$ & 360 \\
\hline 107 & $<600$ & 35,400 & 12,600 & 366 & 1,080 \\
\hline 108 & $<600$ & 47,400 & 21,600 & $\boldsymbol{b}$ & 2,100 \\
\hline 109 & $<600$ & 18,600 & 10,200 & 240 & 1,620 \\
\hline 117 & $<600$ & 3,960 & 1,800 & $\boldsymbol{b}$ & 378 \\
\hline 118 & $<600$ & 6,600 & 2,400 & $\boldsymbol{b}$ & 360 \\
\hline 119 & $<600$ & 174,000 & 114,000 & 1,140 & 1,080 \\
\hline 120 & $<600$ & 7,200 & 6,000 & $b$ & 2,100 \\
\hline 213 & $<600$ & 96,000 & 2,640 & 114 & 66,000 \\
\hline 237 & $<600$ & 18,000 & 7,200 & $b$ & 2,880 \\
\hline 238 & $<600$ & 46,200 & 21,600 & 300 & 2,280 \\
\hline
\end{tabular}

${ }^{a}$ Insufficient activity on smear sample to identify transuranic isotopes.

${ }^{b}$ Not analyzed. 


\section{SIGNIFICANCE OF FINDINGS}

The interior of the Building 7819 Decontamination Facility is grossly contaminated, and the contamination is extremely transferable. The survey team measured high levels of contamination in the northern side of the building, where acid baths and sand blasting were used in the equipment decontamination process, and lower levels in the southern section of the building. Suspected asbestos was observed in the northwest corner of the building. A snakeskin was observed on an overhead heater, indicating the intrusion of wildlife.

Levels of alpha and beta contamination inside the building exceed ORNL guidelines for zoning as a Contamination Area. Gamma exposure rates in several locations in the building exceed ORNL guidelines for zoning as a Radiation Area. Gamma whole-body exposure rates generally ranged from 0.1 to $20 \mathrm{mR} / \mathrm{h}$. Total beta-gamma surface contamination ranged from 1000 to $520,000 \mathrm{dpm} / 100 \mathrm{~cm}^{2}$, and transferable beta-gamma contamination ranged from 200 to $174,000 \mathrm{dpm} / 100 \mathrm{~cm}^{2}$. A pump and a metal table near the pit indicated beta dose rates of $3 \mathrm{rad} / \mathrm{h}$ and $300 \mathrm{mrad} / \mathrm{h}$, respectively, and gamma exposure rates of $250 \mathrm{mR} / \mathrm{h}$ and $25 \mathrm{mR} / \mathrm{h}$ at contact, respectively.

Total alpha contamination levels ranged from 100 to $110,000 \mathrm{dpm} / 100 \mathrm{~cm}^{2}$, and transferable contamination ranged from 21 to $440 \mathrm{dpm} / 100 \mathrm{~cm}^{2}$. The ledge at the north end of the pit above the sink indicated alpha contamination of approximately $100,000 \mathrm{dpm} / 100 \mathrm{~cm}^{2}$. Radionuclide analysis of 11 samples showed the primary beta emitters to be ${ }^{90} \mathrm{Sr}$ and ${ }^{137} \mathrm{Cs}$ with trace amounts of ${ }^{60} \mathrm{Co}$.

The Building 7819 Decontamination Facility was zoned as a Contamination Area and Radiation Area at the time of this survey, and the zoning is certainly justified by the results of the survey. The building will also be zoned as an Airborne Radioactivity Area, based on the results of air sampling. ${ }^{*} A$ minimum of personal protective equipment (PPE) necessary to enter the building should include one pair of coveralls, two pairs of shoe covers, two pairs of gloves, and full-face respirators with HEPA cartridges. The level of PPE may be upgraded, depending on the type of work to be performed inside the building.

The presence of high levels of contamination warrants corrective action measures. Three basic approaches to interim corrective actions are (1) continued isolation of the contaminated area (e.g., roping), including measures to minimize the intrusion of wildlife; (2) removal of contaminated waste, debris, and equipment in the building, to allow a more representative characterization survey without the influence of shine from surrounding objects, and disposal in a designated radioactive waste disposal site; and (3) unplugging of closed drains and resurveying of all drains in the building, followed by replugging of drains to prevent additional contamination of the drainage system.

*M. L. Conner, Office of Radiation Protection, Oak Ridge Natl. Lab., personal communication to K. J. Brown, Health Sciences Research Division, Oak Ridge Natl. Lab., November 1993. 


\section{REFERENCES}

1. RCRA Facilities Assessment-Oak Ridge National Laboratory, ORNL/RAP-12/N1, Martin Marietta Energy Systems, Inc., Oak Ridge Natl. Lab., March 1987.

2. T. E. Myrick, B. A. Berven, W. D. Cottrell, W. A. Goldsmith, and F. F. Haywood, Procedures Manual for the ORNL Radiological Survey Activities (RASA) Program, ORNL/TM-8600, Martin Marietta Energy Systems, Inc., Oak Ridge Natl. Lab., April 1987. 
ORNL/ER-172

\section{DISTRIBUTION}

1. D. T. Bell

2. B. A. Berven

3. H. L. Boston

4-6. K. J. Brown

7. T. W. Burwinkle

8-9. K. Constant

10. M. L. Conner

11. W. D. Cottrell

12. M. F. P. DeLozier

13-15. R. D. Foley

16. H. R. Gaddis

17. S. B. Garland II

18. C. D. Goins, Jr.

19-20. R. C. Gosslee

21. L. Holder, Jr.

22. J. H. Hooyman

23. R. G. Jones

24-26. D. M. Matteo

27. M. E. Murray

28-29. P. T. Owen
30-31. V. P. Patania

32-33. D. E. Rice

34-36. R. E. Rodriguez

37. G. E. Rymer

38. P. A. Schrandt

39. C. S. Sims

40-42. R. E. Swaja

43. M. S. Uziel

44. R. K. White

45-46. J. K. Williams

47. P. S. Wood

48-57. MAD Records Center

58. Central Research Library

59. Technical Library, Y-12

60. Laboratory Records Dept.

61-63. ORNL ER Document Management Center

64-65. Central ER Document Management Center

66. ORNL Patent Section

67. Office of Assistant Manager for Energy Research and Development, DOE Oak Ridge Operations Office, P.O. Box 2001, Oak Ridge, TN 37831-8600

68-69. R. C. Sleeman, DOE Oak Ridge Operations Office, P.O. Box 2001, Oak Ridge, TN 37831-8541

70-71. J. T. Sweeney, DOE Oak Ridge Operations Office, P.O. Box 2001, Oak Ridge, TN 37831-8541

72-73. Office of Scientific and Technical Information, P.O. Box 62, Oak Ridge, TN 37831 

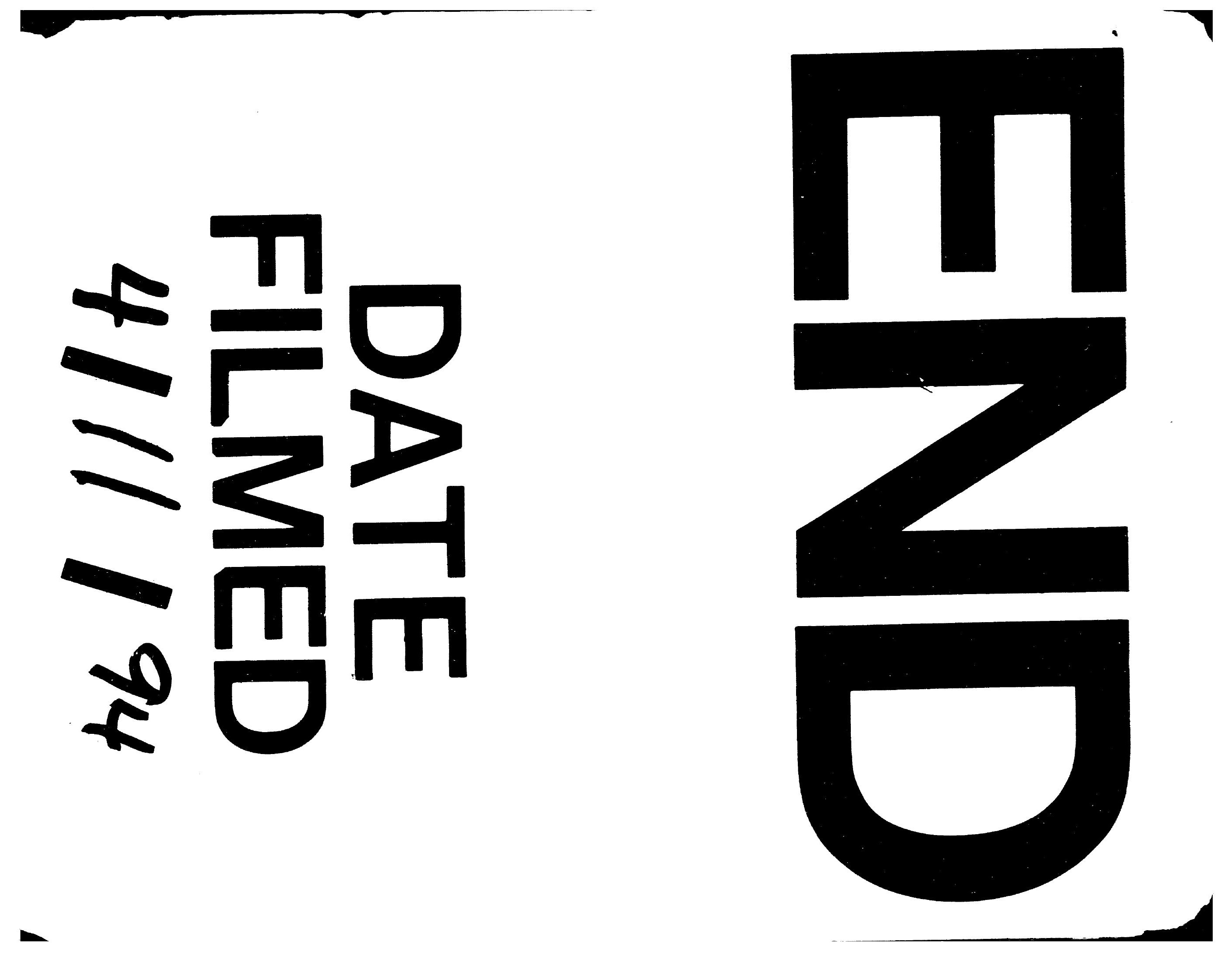
